\title{
Functional BIP: Embedding Connectors in Functional Programming Languages
}

\author{
Romain Edelmann, Simon Bliudze, Joseph Sifakis \\ École polytechnique fédérale de Lausanne \\ Station 14, CH-1015, Lausanne, Switzerland \\ E-mails: 〈name.surname〉@epfl.ch
}

\begin{abstract}
This paper presents a theoretical foundation for functional language implementations of BehaviourInteraction-Priority (BIP). We introduce a set of connector combinators describing synchronisation, data transfer, priorities and dynamicity in a principled way. A static type system ensures the soundness of connector semantics.

Based on this foundation, we implemented BIP as an embedded domain specific language (DSL) in Haskell and Scala. The DSL embedding allows programmers to benefit from the full expressive power of high-level languages. The clear separation of behaviour and coordination inherited from BIP leads to systems that are arguably simpler to maintain and reason about, compared to other approaches.

Keywords: BIP, connectors, combinators, dynamicity, static typing, functional programming, Haskell, Scala
\end{abstract}

\section{Introduction}

When building large concurrent systems, one of the key difficulties lies in coordinating component behaviour and, in particular, management of the access to shared resources of the execution platform. Our approach relies on the BIP framework [1] for component-based design of correct-by-construction applications. BIP provides a simple, but powerful mechanism for the coordination of concurrent components by superposing three layers: Behaviour, Interaction, and Priority. First, component behaviour is described by Labelled Transition Systems (LTS) having transitions labelled with ports and extended with data stored in local variables. Ports form the interface of a component and are used to define its interactions with other components. They can also export part of the local variables, allowing access to the component's data. Second, interaction models, i.e. sets of interactions, define the component coordination. Interactions are sets of ports that define allowed synchronisations between components. Interaction models are specified in a structured manner by using connectors 2. Third, priorities are used to impose scheduling constraints and to resolve 


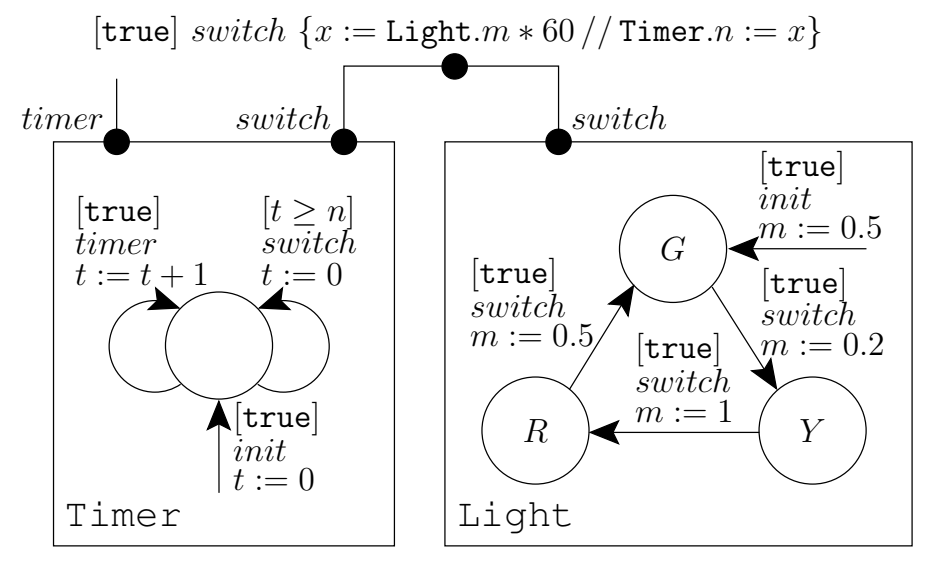

Figure 1: Traffic light in BIP

conflicts when multiple interactions are enabled simultaneously. Interaction and Priority layers are collectively called Glue.

The strict separation between behaviour - i.e. stateful components - and coordination - i.e. stateless connectors and priorities - allows the design of modular systems that are easy to understand, test and maintain. Hierarchical combination of interactions and priorities provides a very expressive coordination mechanism [3, 4. The BIP language has been implemented as a coordination language for $\mathrm{C} / \mathrm{C}++[1$ and Java [5]. It is supported by a tool-set including translators from various programming models into BIP, source-to-source transformers, as well as a number of back-ends for the generation of code executable by dedicated engines 1

Atoms. BIP systems are composed of atoms (atomic components) that have communication ports used for coordination. Atoms have disjoint state spaces; their behaviour is specified as a system of transitions labeled with ports. Figure 1 shows a simple traffic light controller system modelled in BIP. It is composed of two atomic components Timer and Light, modelling, respectively, a timer and the light-switching behaviour. The Timer atom has one state with two self-loop transitions. The incoming arrow, labeled init, denotes the initialisation event. It is guarded by the constant predicate true and has an associated update function $t:=0$, which initialises the data variable $t$, used to keep track of the time spent since the last change of colour. The Light atom determines the colour of the traffic light and the duration (in minutes) that the light must stay in one of the three states, corresponding to the three colours.

Interactions. Interactions between components are defined by hierarchically structured connectors 2, 6]. The system in Fig. 1 has two connectors: a singleton connector with one port timer and no data transfer and a binary connector, synchronising the ports switch of the two components.

\footnotetext{
1 http://www-verimag.imag.fr/Rigorous-Design-of-Component-Based.html
} 
The first, singleton connector is necessary, since, in BIP, only ports that belong to at least one connector can fire. The second connector synchronises the ports Timer.switch and Light.switch; it has an exported (top) port, also called switch, and an associated variable $x$ used for the data transfer. The guard is the constant predicate true, the upward and downward data-flows are defined, respectively, by the assignments $x:=$ Light. $m * 60$ and Timer. $n:=x$. Thus, upon each synchronisation, Light informs Timer about the amount of time to spend in the next location, converting it from minutes to seconds.

Connectors define sets of interactions based on the synchronisation attributes of the connected ports, which may be either trigger or synchron (Fig. 2 $)$. If all connected ports are synchrons, then synchronisation is by rendezvous, i.e. the defined interaction may be executed only if all the connected components allow the transitions of those ports (Fig. 2 b). If a connector has at least one trigger, the synchronisation is by broadcast $t^{2}$ i.e. the allowed interactions are all non-empty subsets of the connected ports comprising at least one of the trigger ports (Fig. 2 ). More complex connectors can be built hierarchically (Fig. 2e).

In the textual notation used for the Algebra of Connectors in [2, triggers are marked by primes. Thus, the three connectors in Fig. 2 (from top to bottom) are written, respectively, as $a b c, a^{\prime} b c$ and $a^{\prime} b^{\prime} c$. In hierarchical connectors, square brackets are used to denote sub-connectors. Thus, the three connectors in Fig. 2k (from left to right) are written, respectively, as $a[b c], a^{\prime}[b c]$ and $a^{\prime}\left[b^{\prime} c\right]$.

Notice that, $a b c$ and $a[b c]$ are equivalent, i.e. define the same set of interactions (here the singleton set $\{a b c\})$, whereas $a^{\prime} b c$ and $a^{\prime}[b c]$ are not equivalent, defining, respectively, the interaction sets $\{a, a b, a c, a b c\}$ and $\{a, a b c\}$. Notice that this equivalence relation is not a congruence. Indeed, although connectors $a b c$ and $a[b c]$ are equivalent, this is not the case for $a b c d^{\prime}$ and $a[b c] d^{\prime}$, since the latter does not allow the interactions involving only one of the ports $b$ and $d$, i.e. $b d, c d, a b d$ and $a c d$. A sound and complete axiomatisation is provided in [2. In particular, it is shown that, if two connectors $\gamma_{1}$ and $\gamma_{2}$ are equivalent, then $\left[\gamma_{1}\right]$ and $\left[\gamma_{2}\right]$ are congruent, i.e. either of them, used as a sub-connector of a larger one, can be substituted by the other.

The description of each (sub-)connector consists of three parts:

1. A Boolean guard determining the enabledness of each interaction allowed by the connector, depending on the values of the provided data: an interaction is only enabled if the data provided by the components satisfies the guard [6].

2. A control part specifying a relation between a set of bottom ports and a single top port. In hierarchical connectors, sub-connector top ports are used as higher-level bottom ports. Each

\footnotetext{
2 Although we use the term "broadcast" by analogy with message passing-trigger ports initialise interactions, whereas synchrons join if they are enabled-, connectors synchronise ports, i.e. no messages passing is involved.
} 
(a) Port use (b) Flat Connectors

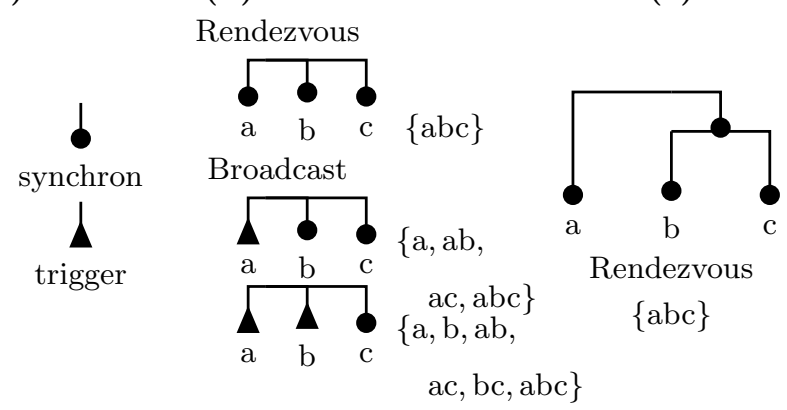

(c) Hierarchical Connectors
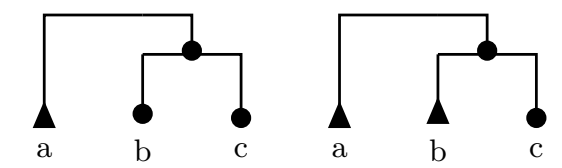

Atomic broadcast Causality chain

$\{\mathrm{a}, \mathrm{abc}\}$ $\{\mathrm{a}, \mathrm{ab}, \mathrm{abc}\}$

Figure 2: Flat and hierarchical BIP connectors

port of an atom can be used in several connectors. However, a connector can involve at most one port per atomic component. The same applies to top ports of connectors.

3. A data-flow part specifying the computation associated with each interaction. The computation can affect variables associated with the ports. It consists of an upstream computation followed by a downstream computation. The former is specified by a function that takes as arguments the values exported through the ports involved in the interaction. The computed value is exported through the top port. The downstream computation produces values associated with the synchronised ports from the value received at the top port. This allows bidirectional exchange of information upon synchronisations among components.

Priorities. Finally, priorities are used to impose scheduling constraints and to resolve conflicts when multiple interactions are enabled simultaneously. Interaction and Priority layers are collectively called Glue.

For instance, notice that, when $t \geq n$, both transitions, timer and switch, of the Timer atom in Fig. 1 are enabled. Since all other guards in the system are constant predicates true, this means that both connectors can fire. Imposing the priority timer < switch resolves this choice, so that switching is performed whenever possible. In general, it is not necessary to impose priorities in all conflict situations: according to the BIP semantics, one of the enabled maximal priority interactions is chosen non-deterministically [2].

Although, the theoretical presentation in 2] completely separates interactions and priorities, in all practical applications and BIP implementations, one always implicitly assumes maximal progress, which defines the priority $\alpha<\beta$ for two interactions allowed by the same connector, provided that the inclusion $\alpha \subset \beta$ holds on $\alpha$ and $\beta$ considered as sets of ports. Thus, among the interactions defined by the connector $a^{\prime} b c(\overline{\text { Fig. } 2}$ b), the priority relations $a<a b<a b c$ and $a<a c<a b c$ are always enforced in all practical applications. Unless additional priorities are provided explicitly, interactions $a b$ and $a c$ are incomparable. Thus, when all three ports $a, b$ and $c$ 
are enabled, but the interaction $a b c$ is disabled because of a data guard, one of the interactions $a b$ and $a c$ will be chosen non-deterministically.

This paper adapts the BIP coordination mechanisms to the context of functional programming languages: systems are composed of atoms, ports and a single static connector. As in the original BIP implementations, atoms have their own private memory, which is not shared with other atoms. Thus, atoms can communicate only by interacting through the ports of the system.

There are three main differences between Functional BIP and the original implementations. First of all, Functional BIP abandons code generation, introducing instead the primitives necessary to programmatically describe both the atomic behaviour and the glue. In particular, ports offer a very simple interface which consists of a single operation, called await. When invoking await, the atom sends a value to the port and blocks until the port sends a value back to the atom. Atoms may wait on multiple ports simultaneously. Ports are typed to describe the type of values that can be sent and received through them.

The second - most important-difference is that, in Functional BIP, ports are not directly associated with atoms. On the contrary, they are globally accessible entities used for communication and coordination. We introduce the combinator Bind to attach a port to a specific atom. Such dissociation of ports from atoms allows us to introduce forms of dynamicity, which are not currently available in any of the original BIP implementations: some atoms are created at the system initiation, while others may be spawned by existing atoms at run time; we introduce the combinator Dynamic, which binds all existing atoms, including those created at run time, to a given port.

Finally, as mentioned above, a Functional BIP system comprises only one connector, as opposed to a set of connectors in the original BIP approach. Notice, however, that this does not reduce the expressiveness of the BIP coordinating mechanism, since any number of connectors can be combined into a single one using the OneOf combinator (see Sect. 3.1.3). The connector of a system links atom-port pairs to define the possible interactions. The connector is unique and fixed throughout the system lifespan.

We introduce a number of combinators to describe connectors. Connector combinators are used to hierarchically build complex connectors, starting from a set of basic ones [7]. Figure 3 shows the Functional BIP representation of the switch connector from the example in Fig. 1. This is realised using two combinators: Both0f (see Sect. 3.1.4 and Mapped (see Sect. 3.3.1). The former synchronises the switch ports of the two atoms, whereas the latter is used to convert the data value sent by the first atom from minutes to seconds.

In Functional BIP, the connector is represented as a tree, with port-atom bindings at the leaves and combinators in the nodes. The arity of each node depends on the corresponding combinator.

Data transfer within the connector occurs in two phases: upward and downward. During the 


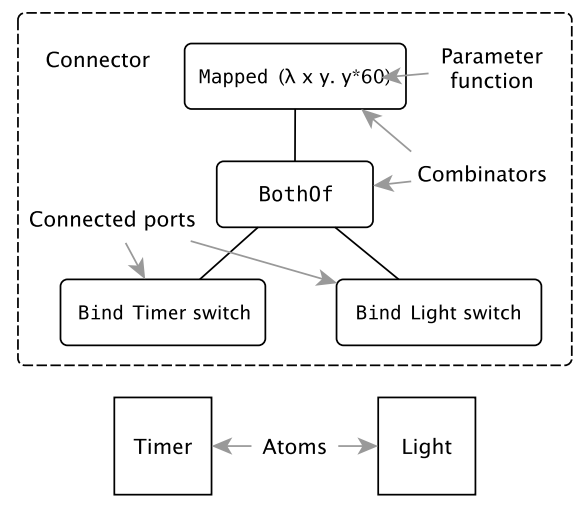

Figure 3: A Functional BIP view of the switch connector from the example in Fig. 1

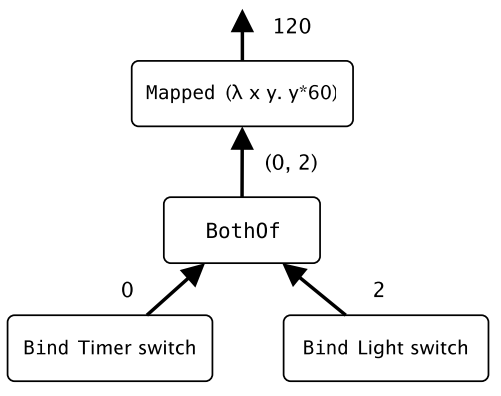

(a) Upward phase

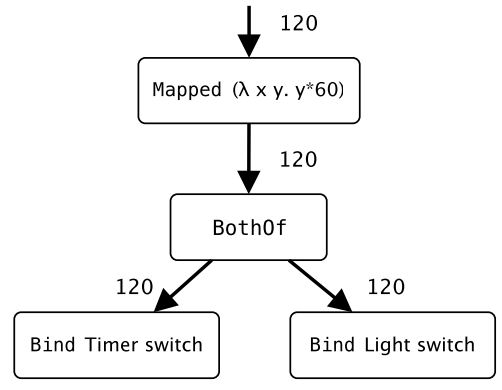

(b) Downward phase

Figure 4: Data transfer within connectors

upward phase, the values sent through the ports by the atoms are collected and propagated up through the connector hierarchy. Once a value reaches the top of the hierarchy, it is transferred downward, back to the atoms involved in the interaction. Both up- and downward data transfers at each node of the tree are defined by the corresponding combinator. Figure 4 illustrates the data transfer within the connector in Fig. 3, assuming that the atoms Timer and Light have sent, respectively, the values 2 and 0 on the port switch.

A connector ensures that atoms may only receive a value on the ports on which they are waiting. To maintain this invariant, we ensure that only the combinators which provided a value during the upward computation phase may receive a value during the downward phase.

The listings in Fig. 5 and Fig. 6 show Haskell and Scala implementations of the system presented in Fig. 1 using the Functional BIP framework.

The main contributions of this paper are the following:

- We present a set of combinators to build connectors which can describe synchronisation, data 


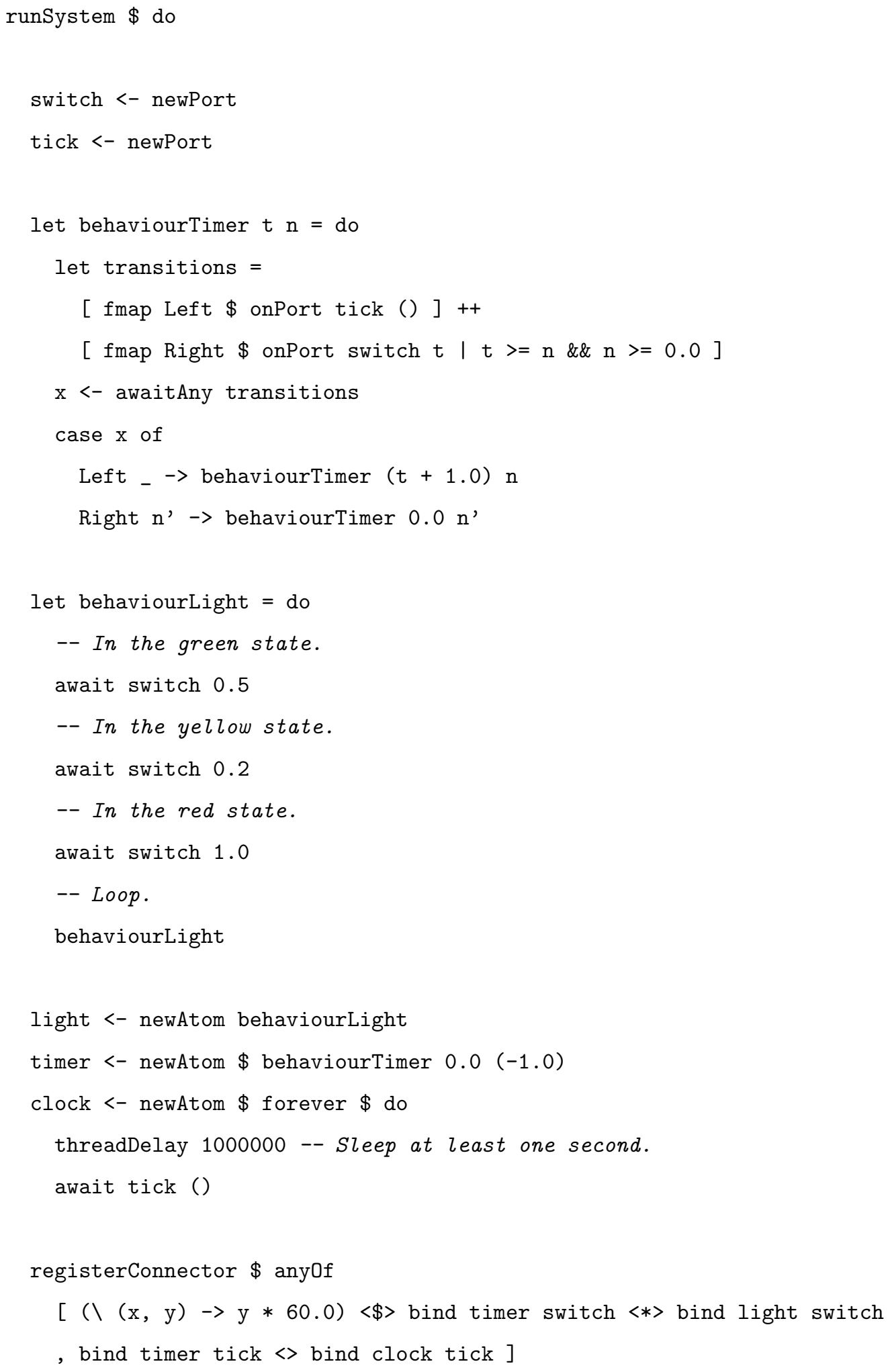

Figure 5: Implementation in Haskell of the example from Fig. 3 


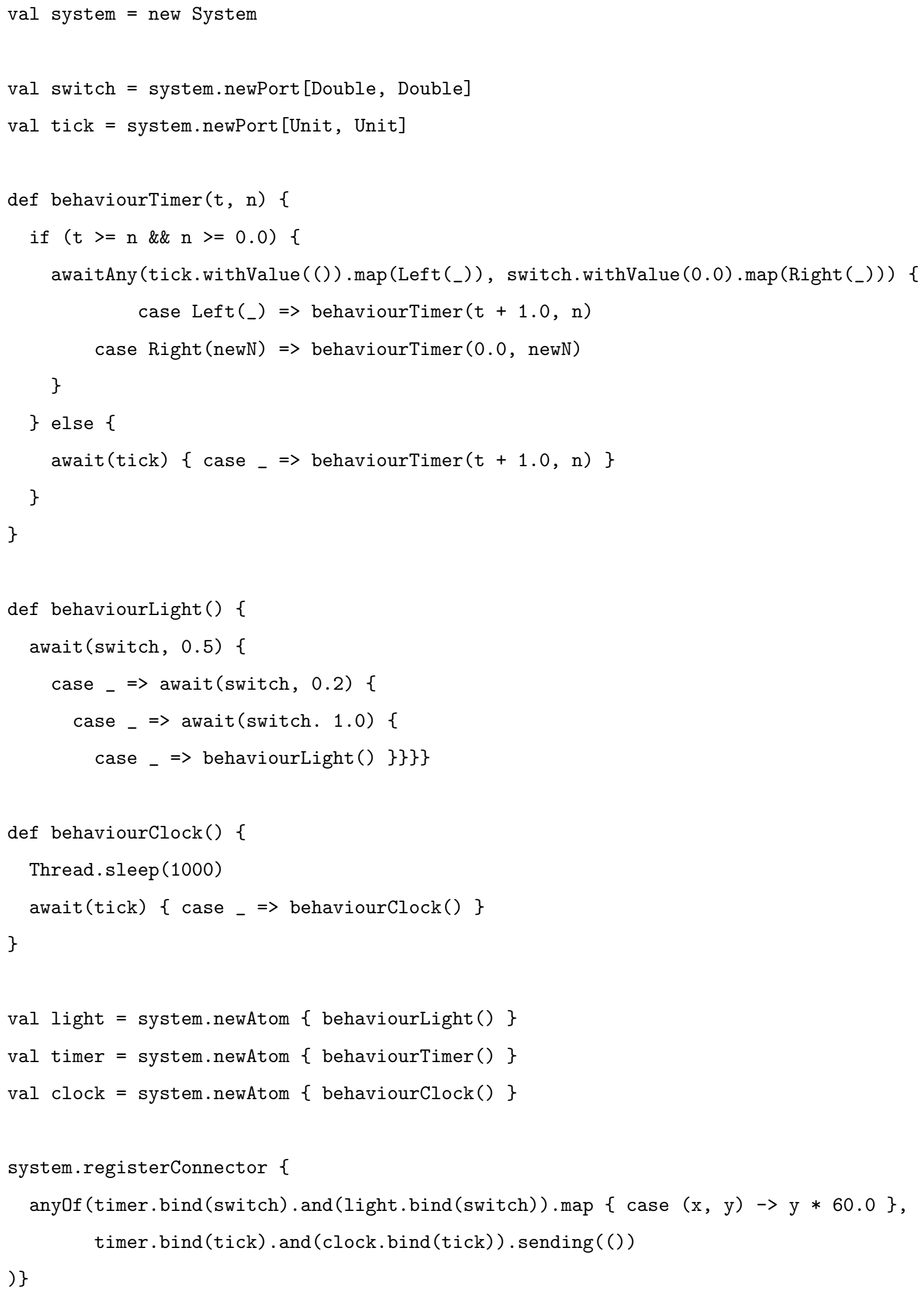

Figure 6: Implementation in Scala of the example from Fig. 3 
transfer, priorities and dynamicity. We introduce the formal semantics and typing rules of those combinators and present some of their algebraic properties.

- We present implementations of the concepts developed in this paper in two functional programming languages, Haskell [8] and Scala [9]. In each case, we show how the concepts can be transposed using well known idioms of the language. The two resulting frameworks are released under open source licenses and are freely available for download and us $\mathrm{f}^{3}$.

This paper is based on [10. A brief announcement was made at the Interaction and Concurrency Experience workshop [11].

The rest of this paper is structured as follows. Section 2 introduces the typing and semantic framework for connector combinators. Section 3 introduces the core, priority, data and dynamic connector combinators. Section 4 discusses their algebraic properties. Section 5 describes an implementation of the presented concepts in Haskell and Scala. Section 6 discusses the related work. Section 7 concludes and summarises the paper.

\section{Semantic Framework}

Before we introduce the connector combinators, we introduce notation and concepts that will be used to describe their semantics and types.

\subsection{Partial Functions}

We denote by $A \rightarrow B$ the set of partial functions from the set $A$ to the set $B$. We will sometimes use the fact that partial functions can be represented as sets of pairs from $A \times B$, in particular when we will construct such partial functions. For instance, we will denote the empty partial function by $\emptyset$ and the singleton partial function that maps $x$ to $y$ by $\{x \mapsto y\}$.

\subsection{Universe of Values}

We denote by $\mathcal{V}$ the set of values which can be handled and exchanged by the atoms. We assume that this set contains at least the Boolean values true and false, integers, all pairs of values and all total functions from values to values. Connectors are also part of the universe of values.

\footnotetext{
3 Haskell version: https://github.com/redelmann/bip-in-haskell

Scala version: https://github.com/redelmann/bip-in-scala
} 


\subsection{Types}

The values are equipped with a polymorphic type system, similar to Haskell's type system. We denote by $\mathcal{T}$ the set of all types and by $v: t$ (respectively $x: t$ ) the predicate "value (respectively variable) $v \in \mathcal{V}$ has type $t \in \mathcal{T}$ ". For each type $t \in T$, we denote by $\operatorname{sem}(t) \subseteq \mathcal{V}$ its semantic domain, i.e. the set of values ranged over by variables $x: t$. Booleans are given the type bool, integers the type int while pairs of values of type $a$ and $b$ are given the type $a \times b$. Total functions from values of type $a$ to values of type $b$ are given the type $a \rightarrow b$. Depending on the context, we will use both the "type" notation, $f: t_{1} \rightarrow t_{2}$, and the "set" notation, $f: \operatorname{sem}\left(t_{1}\right) \rightarrow \operatorname{sem}\left(t_{2}\right)$. The main difference is that, without the function name, $t_{1} \rightarrow t_{2}$ denotes the type, whereas $\operatorname{sem}\left(t_{1}\right) \rightarrow \operatorname{sem}\left(t_{2}\right)$ denotes the set of functions of this type, i.e. $\operatorname{sem}\left(t_{1} \rightarrow t_{2}\right)=\operatorname{sem}\left(t_{1}\right) \rightarrow \operatorname{sem}\left(t_{2}\right)$.

Connectors propagating values of type $u$ during the upward phase and receiving values of type $d$ during the downward phase have the type $u \uparrow \downarrow d$. The type system dictates how the connector combinators can be used.

\subsection{Atoms}

We denote by $\mathcal{A}$ the set of atom identifiers of a system.

\subsection{Ports}

We denote by $P$ the set of ports of a system. Each port $p \in P$ is associated with two types: sendType $(p)$ and receiveType $(p)$. Atoms can only send values of type sendType $(p)$ through the port $p$ and are guaranteed that the value eventually received from the port, if any, has type receiveType $(p)$.

\subsection{Connectors}

We denote by $\mathcal{C}$ the set of connectors $c$ for which there exists at least two types $u$ and $d$ such that $c: u \uparrow \downarrow d$.

\subsection{System States}

A system state is as partial function, mapping atom-port pairs to corresponding values sent by the atom through the port. The set of states is:

$$
\mathcal{S}=\{f \in(\mathcal{A} \times P) \rightarrow \mathcal{V} \mid \forall((a, p) \mapsto x) \in f, x: \operatorname{sendType}(p)\}
$$

In a given system state, all atoms are waiting on a (possibly empty) subset of ports. When a port is active for an atom, a value of the appropriate type has been sent through it. Figure 7 shows a system state where atoms $a_{i}$ have sent values $v_{i}$ on ports $p_{i}$. 


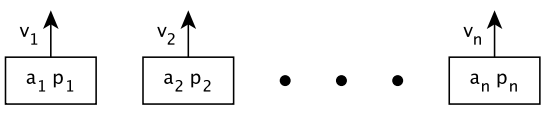

Figure 7: System state

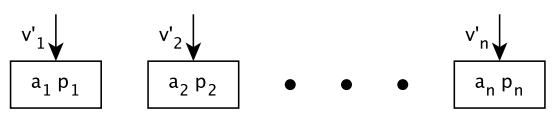

Figure 8: Assignment

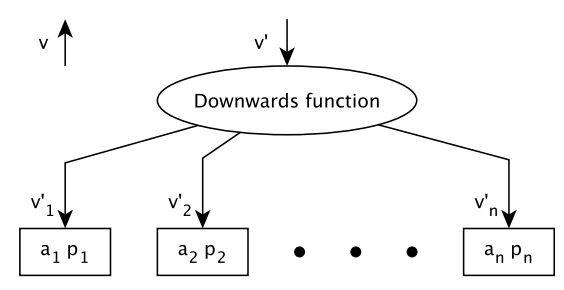

Figure 9: Open interaction

\subsection{Assignments}

Similarly, an assignment is a partial function, mapping atom-port pairs to corresponding values received by the atom on the port. The set of assignments is:

$$
\mathcal{R}=\{f \in(\mathcal{A} \times P) \rightarrow \mathcal{V} \mid \forall((a, p) \mapsto x) \in f, x: \text { receiveType }(p)\} .
$$

An assignment maps each atom-port pair to at most one value. The value assigned, if any, is the value received by the atom on the given port. Figure 8 represents an assignment where atoms $a_{i}$ receive values $v_{i}$ on the ports $p_{i}$.

\subsection{Open Interactions}

We denote by $\mathcal{O}=\mathcal{V} \times \bigcup_{u \in \mathcal{T}}(\operatorname{sem}(u) \rightarrow \mathcal{R})$ the set of open interactions. An open interaction consists of an upward value in $\mathcal{V}$ and a downward function in $\operatorname{sem}(u) \rightarrow \mathcal{R}$ that, given a downward value of some type $u$ returns an assignment. Those interactions are called open as the values exchanged are exposed. Figure 9 shows the schematic representation of an open interaction.

When the upward value is in the domain of the downward function, the open interaction can be closed to obtain a valid assignment. In this case, the upward value is used as a downward value:

$$
\begin{aligned}
& \text { close }: \mathcal{O} \rightarrow \mathcal{R} \\
& \operatorname{close}((v, f))=f(v), \text { if } v \in \operatorname{domain}(f) .
\end{aligned}
$$

\subsection{Downward Compatibility}

We consider two assignments $R_{1}, R_{2} \in \mathcal{R}$ to be downward compatible if and only if:

$$
\left\{a \mid \exists p \in P .(a, p) \in \operatorname{domain}\left(R_{1}\right)\right\} \cap\left\{a \mid \exists p \in P .(a, p) \in \operatorname{domain}\left(R_{2}\right)\right\}=\emptyset .
$$


Intuitively, two assignments are downward compatible if they involve distinct sets of atoms. We extend this notion to downward functions. Downward functions $f_{1}, f_{2}$ are downward compatible if and only if they produce downward compatible assignments for all possible downward values. Notice that, if two assignments $R_{1}, R_{2} \in \mathcal{R}$ are downward compatible, then $R_{1} \cup R_{2}$ is also an assignment. Indeed, downward compatibility ensures that two interactions can be fused [2] without violating the requirement that atoms are only involved once in an interaction. For two downward compatible functions, $f_{1}, f_{2}: \operatorname{sem}(u) \rightarrow \mathcal{R}$, we will denote $f_{1} \cup f_{2}: \operatorname{sem}(u) \rightarrow \mathcal{R}$ the downward function defined by $\left(f_{1} \cup f_{2}\right)(v) \stackrel{\text { def }}{=} f_{1}(v) \cup f_{2}(v)$.

\section{Connector Combinators}

Using the semantic framework introduced in Sect. 2, we can now introduce the set of combinators used to describe connectors. For the sake of clarity, we will introduce combinators progressively, in related groups. First, we introduce core combinators, which correspond to the Algebra of Interactions [2]. Then, we introduce combinators describing priorities, data manipulation and dynamicity.

For each connector combinator, we will provide the type inference rule defining the type of the resulting connector from the types of the parameters and children connectors. We also provide formal semantics of each combinator. To this end, we define the following semantic function:

$$
[\cdot]: \mathcal{C} \rightarrow \mathcal{S} \rightarrow 2^{\mathcal{O}}
$$

This semantic function gives, for each connector and system state, the set of possible open interactions. We will define this function progressively as we encounter the different set of connector combinators. We will define it recursively for each of the possible combinators.

\subsection{Core Combinators}

\subsubsection{Bind}

This combinator takes a port and an atom and binds them together. It is equivalent to the port of an atomic component in BIP. The type of the resulting connector depends on the send and receive types of the port:

$$
\text { (Bind a } p): \operatorname{sendType}(p) \uparrow \downarrow \text { receive Type }(p)
$$

The resulting connector provides a single interaction if the atom is currently waiting on the port and none otherwise. The value propagated upward by this connector is the value, if any, that was sent through the port by the atom. When this connector receives a value during the downward 
phase, it is transmitted to the atom via the given port. Expressed in terms of the open semantics function, the behaviour of the resulting connector is defined as:

$$
[\text { Bind } a p](S)= \begin{cases}\{(S(a, p),\{v \mapsto\{(a, p) \mapsto v\} \mid v: \text { receiveType }(p)\})\}, & \text { if }(a, p) \in \operatorname{domain}(S), \\ \emptyset, & \text { otherwise. }\end{cases}
$$

If the system state has an entry for the given atom-port pair, then a single open interaction is possible. The upward value of the open interaction is the value sent by the port, while the downward function propagates the downward value to the atom and port.

\subsubsection{Success and Failure}

These two combinators do not involve any atoms and ports. They have arity zero and can only be found at the leaves of the connector tree.

Regardless of the system state, Success $v$ always provides a single open interaction, whose upward value is $v$. Failure, on the other hand, represents a connector that is never enabled. While by themselves, these combinators do not present any practical interest, they can be combined with others to build more useful connectors. They correspond to the elements 1 and 0 of the Algebra of Interactions [2]. The types of connectors defined by Success and Failure are given by the following inference rules:

$$
\frac{v: u}{(\text { Success } v): u \uparrow \downarrow d}, \quad \frac{}{\text { Failure }: u \uparrow \downarrow d} .
$$

Notice that Success is polymorphic in the downward type and Failure in both the upward and downward types. Let us denote $\operatorname{Success}{ }^{u \uparrow \downarrow d} v$, with $v: u$, the instantiation of Success $v$ for the type $u \uparrow \downarrow d$. The open-interaction semantics of the two connectors is defined as follows:

$$
\begin{aligned}
\left.\operatorname{SSuccess}^{u \uparrow \downarrow d} v\right](S) & =\{(v,\{w \mapsto \emptyset \mid w \in \operatorname{sem}(d)\})\}, \\
{[\text { Failure }](S) } & =\emptyset .
\end{aligned}
$$

\subsubsection{OneOf}

This combinator expresses the non-deterministic choice between two connectors. The connector OneOf $c_{1} c_{2}$ behaves as either $c_{1}$ or $c_{2}$. This combinator corresponds to the union operation in the Algebra of Interactions [2]. The type and open interaction semantics of OneOf $c_{1} c_{2}$ are defined as follows:

$$
\begin{gathered}
\frac{c_{1}: u \uparrow \downarrow d \quad c_{2}: u \uparrow \downarrow d}{\left(\text { OneOf } c_{1} c_{2}\right): u \uparrow \downarrow d}, \\
{\left[\text { OneOf } c_{1} c_{2}\right](S)=\left[c_{1}\right](S) \cup\left[c_{2}\right](S) .}
\end{gathered}
$$

Thus, the interactions possible in this connector are all interactions that are possible in either $c_{1}$ or $c_{2}$. 


\subsubsection{Bothof}

This last core combinator represents the fusion of two connectors. It corresponds to the fusion operator in the Algebra of Interactions [2]. The corresponding type inference rule is

$$
\frac{c_{1}: u_{1} \uparrow \downarrow d \quad c_{2}: u_{2} \uparrow \downarrow d}{\left(\text { BothOf } c_{1} c_{2}\right):\left(u_{1} \times u_{2}\right) \uparrow \downarrow d} .
$$

Thus, Both0f $c_{1} c_{2}$ propagates upward the pair of upward values coming from $c_{1}$ and $c_{2}$. In the presence of non-determinism, this connector returns all possible combinations of interactions from $c_{1}$ and $c_{2}$ :

$$
\begin{aligned}
{\left[\text { Both0f } c_{1} c_{2}\right](S)=\left\{\left(\left(x_{1}, x_{2}\right), f_{1} \cup f_{2}\right) \mid\left(x_{1}, f_{1}\right) \in\left[c_{1}\right](S),\left(x_{2}, f_{2}\right) \in\left[c_{2}\right](S)\right. \text { and }} \\
\left.f_{1} \text { and } f_{2} \text { are downward compatible }\right\} .
\end{aligned}
$$

Interactions that would lead to atoms receiving more than one value during the downward phase are filtered out by the downward compatibility check.

\subsubsection{Examples}

Example 3.1. The Optional combinator, which takes as parameters a value $v$ and a connector $c$, and returns a connector that provides any interaction provided by $c$, plus an extra interaction whose upward value is $v$, is derived as follows:

$$
\text { Optional } v c=\text { OneOf } c(\operatorname{Success} v) \text {. }
$$

Note that the extra interaction introduced by this combinator does not involve any atoms.

Example 3.2. The AnyOf and Allof combinators, which generalise, respectively, the OneOf and the BothOf combinators to an arbitrary number of underlying connectors, are recursively built as follows:

$$
\begin{aligned}
\text { AnyOf }<> & =\text { Failure } \\
\text { AnyOf }\left\langle c_{1}, \ldots>\right. & =\text { OneOf } c_{1}(\text { AnyOf }<\ldots>)
\end{aligned}
$$

and

$$
\begin{aligned}
\text { Allof }<> & =\text { Success }<> \\
\text { Allof }<c_{1}, \ldots> & =\text { Both0f } c_{1}(\text { Allof }<\ldots>) .
\end{aligned}
$$

The Allof combinator allows us to define an arbitrary rendezvous connector as shown in Fig. 2 p. The following example shows how to define broadcast connectors. 
Example 3.3. Recall the textual notation of the Algebra of Connectors (Sect. 1) and consider a connector $\left[t_{1}\right]^{\prime} \ldots\left[t_{n}\right]^{\prime}\left[s_{1}\right] \ldots\left[s_{m}\right]$ with $n$ triggers and $m$ synchrons. It is easy to see [2] that this connector can be equivalently rewritten as $\left[\left[t_{1}\right]^{\prime} \ldots\left[t_{n}\right]^{\prime}\right]^{\prime}\left[\left[s_{1}\right]^{\prime} \ldots\left[s_{m}\right]^{\prime}\right]$.

We define the following derived combinators:

$$
\begin{aligned}
& \text { Trigger } t s=\text { OneOf }(\text { BothOf } t s) t, \\
& \text { ManyOf } c_{1} c_{2}=\text { OneOf }\left(\text { BothOf } c_{1} c_{2}\right)\left(\text { OneOf } c_{1} c_{2}\right), \\
& \text { ManyOf } c_{1} c_{2}<\cdots>=\text { ManyOf } c_{1}\left(\text { ManyOf } c_{2}<\cdots>\right),
\end{aligned}
$$

which allow us to encode arbitrary broadcast connectors $\left[t_{1}\right]^{\prime} \ldots\left[t_{n}\right]^{\prime}\left[s_{1}\right] \ldots\left[s_{m}\right]$ as follows:

$$
\text { Broadcast }\left(t_{1} \ldots t_{n}\right)\left(s_{1} \ldots s_{m}\right)=\operatorname{Trigger}\left(\text { ManyOf } t_{1} \ldots t_{n}\right)\left(\text { ManyOf } s_{1} \ldots s_{m}\right) \text {. }
$$

\subsection{Priority Combinators}

In this sub-section, we introduce the combinators used to specify priorities. Priorities are used to inhibit the execution of certain interactions when interactions of higher priority are possible. Contrary to the classical BIP syntax, where priorities are defined by a separate syntactic construction and can be applied only at the top level of a connector within a compound component, priority combinators can be applied at any level in the connector hierarchy.

\subsubsection{Firstof}

This combinator imposes fixed-order priority among the sub-connectors. In the connector Firstof $c_{1} c_{2}$, interactions from $c_{1}$ will be preferred over interactions from $c_{2}$, whenever the former are available. The type and semantics of the resulting connector are defined as follows

$$
\frac{c_{1}: u \uparrow \downarrow d \quad c_{2}: u \uparrow \downarrow d}{\left(\text { First0f } c_{1} \quad c_{2}\right): u \uparrow \downarrow d},
$$

and

$$
\text { [Firstof } \left.c_{1} c_{2}\right](S)= \begin{cases}{\left[c_{1}\right](S),} & \text { if }\left[c_{1}\right](S) \neq \emptyset \\ {\left[c_{2}\right](S),} & \text { otherwise }\end{cases}
$$

\subsubsection{Maximal}

Given a partial ordering on the upward data domain encoded by a predicate $g$, the connector Maximal $g c$ returns all interactions from the connector $c$ whose upward values are maximal. The parameter function $g$ should return true when its first parameter is strictly less than its second parameter, and false otherwise. The type of the resulting connector is defined by the inference rule

$$
\frac{g:(u \times u) \rightarrow \text { bool } \quad c: u \uparrow \downarrow d}{(\text { Maximal } g c): u \uparrow \downarrow d}
$$


whereas its semantics is given by

$$
\text { [Maximal } g c](S)=\left\{(x, f) \in[c](S) \mid \nexists\left(x_{1}, f_{1}\right) \in[c](S) . g\left(x, x_{1}\right)=\text { true }\right\}
$$

\subsubsection{Examples}

Example 3.4. Figure 10 presents a system of two agents, $A_{1}$ and $A_{2}$, whose behaviour is given as automata. The connector ensures that only one of $A_{1}$ and $A_{2}$ may be in the state work at the same time.

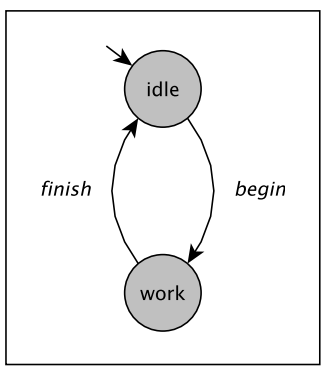

(a) The behaviour of atoms

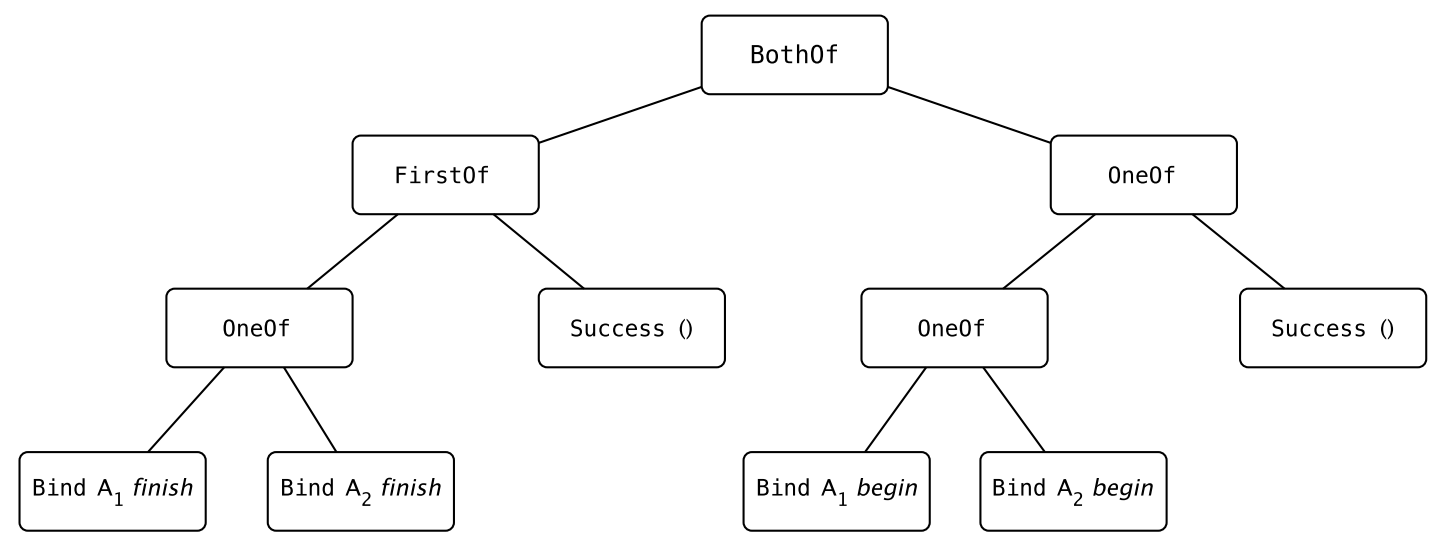

(b) The connector

Figure 10: Mutual exclusion using Firstof

The use of First0f in this example ensures that the finish transitions are taken as soon as possible. If the OneOf combinator was used instead, the mutual exclusion property could be violated, as the connector would allow begin transitions to fire alone.

To see that more concretely, let us examine the different cases where the mutual exclusion property could potentially be violated:

1. Atoms $A_{1}$ and $A_{2}$ are both waiting on the port begin. In this case, the connector ensures that only one of the atoms may take the transition and enter the critical section. As One0f 
may only select one of the underlying connector, not both begin transitions may be part of the same interaction.

2. $A_{1}$ is waiting on the finish port and $A_{2}$ on the begin port. A problem would occur if $A_{2}$ entered the work state while $A_{1}$ stayed in the same state. The use of Firstof within the connector ensures that $A_{1}$ takes a transition from the work state to the idle state, thereby avoiding the problematic situation.

3. The case when $A_{2}$ is waiting on the finish port and $A_{1}$ on the begin port is symmetrical to the previous case.

Thus, since in the initial state both atoms are idle, they cannot end up being in the work state simultaneously, i.e. the mutual exclusion property is, indeed, ensured.

Example 3.5. In Example 3.3, we have shown how to encode arbitrary broadcast connectors using the core combinators. In order to implement the maximal progress assumption, we replace two of the occurrences of OneOf in (12) and (13) by First0f:

$$
\begin{aligned}
& \text { TriggerMP } t s=\text { FirstOf }(\text { BothOf } t s) t, \\
& \text { ManyOfMP } c_{1} c_{2}=\text { FirstOf }\left(\text { BothOf } c_{1} c_{2}\right)\left(\text { OneOf } c_{1} c_{2}\right), \\
& \text { ManyOfMP } c_{1} c_{2}<\cdots>=\text { ManyOfMP } c_{1}\left(\text { ManyOfMP } c_{2}<\cdots>\right) .
\end{aligned}
$$

The definition of the broadcast combinator stays the same:

$$
\text { BroadcastMP }\left(t_{1} \ldots t_{n}\right)\left(s_{1} \ldots s_{m}\right)=\operatorname{TriggerMP}\left(\operatorname{ManyOfMP} t_{1} \ldots t_{n}\right)\left(\operatorname{ManyOfMP} s_{1} \ldots s_{m}\right) \text {. }
$$

\subsection{Data Combinators}

We now introduce the combinators for data manipulation in the connectors.

\subsubsection{Mapped and ContraMapped}

These two combinators apply a parameter function to, respectively, the upward and downward values propagated by the underlying connector. The types of the resulting connectors are defined by the inference rules:

$$
\frac{g: v \rightarrow u \quad c: v \uparrow \downarrow d}{(\text { Mapped } g c): u \uparrow \downarrow d}, \quad \frac{g: d \rightarrow e \quad c: u \uparrow \downarrow e}{\text { (ContraMapped } g c): u \uparrow \downarrow d} .
$$

The two combinators never modify the number of possible interactions:

$$
\begin{aligned}
\text { [Mapped } g c](S) & =\{(g(x), f) \mid(x, f) \in[c](S)\}, \\
\text { ContraMapped } g c](S) & =\{(x, f \circ g) \mid(x, f) \in[c](S)\} .
\end{aligned}
$$




\subsubsection{Guarded}

This combinator is used to filter out open interactions whose upward values fail to satisfy a predicate passed as the argument. The connector type is defined by the inference rule

$$
\frac{g: u \rightarrow \text { bool } \quad c: u \uparrow \downarrow d}{(\text { Guarded } g c): u \uparrow \downarrow d} .
$$

This combinator allows restricting non-determinism, ensuring that upward values all satisfy a given predicate:

$$
[\text { Guarded } g c](S)=\{(x, f) \in[c](S) \mid g(x)=\text { true }\}
$$

\subsubsection{Feedback}

This last data manipulation combinator allows feeding the upward value back into the downward propagation phase. The typing inference rule and the semantics are defined by putting

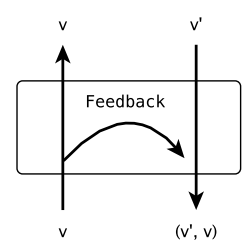

$$
\begin{gathered}
\frac{c: u \uparrow \downarrow(d \times u)}{(\text { Feedback } c): u \uparrow \downarrow d}, \\
{[\text { Feedback } c](S)=\left\{\left(x, f \circ \operatorname{tag}_{x}\right) \mid(x, f) \in[c](S)\right\},}
\end{gathered}
$$

where $\operatorname{tag}_{x}(y) \stackrel{\text { def }}{=}(y, x)$ (see the figure on the left).

\subsubsection{Examples}

Example 3.6. Consider the connector in Fig. 11. This connector synchronises two atoms, $A_{1}$ and $A_{2}$, on the exchange port. The value sent back to each atom is the value that was sent by the other atom.

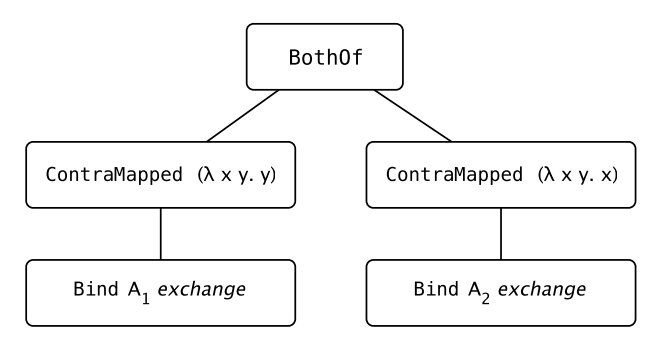

Figure 11: Example use of ContraMapped

Example 3.7. The ability to manipulate data allows us to encode First0f by combining Mapped, OneOf and Maximal:

$$
\text { FirstOf } \left.c_{1} c_{2}=\text { Mapped untag (Maximal } c m p_{2}\left(\text { OneOf }\left(\text { Mapped } \operatorname{tag}_{2} c_{1}\right)\left(\text { Mapped } \operatorname{tag}_{1} c_{2}\right)\right)\right)
$$


where the function untag, $c m p_{2}$ and $t a g_{n}$ are defined as by putting

$$
\begin{aligned}
\operatorname{untag}(x, n) & =x, \\
\operatorname{cmp}_{2}((x, n),(y, m)) & =n<m, \\
\operatorname{tag}_{n}(x) & =(x, n) .
\end{aligned}
$$

Example 3.8. In Examples 3.3 and 3.5, we have shown how one can encode arbitrary broadcast connectors with Example 3.3 and without Example 3.5 the maximal progress assumption. However, both of these encodings potentially incur a huge overhead at run time, due to the duplication of operand connectors in (12) and (13). We now provide an alternative, efficient encoding of broadcast without maximal progress. To this end, we define the following derived combinators (denoting by $c s, t s$ and $s s$, respectively, the lists of connectors, triggers and synchrons):

$$
\begin{aligned}
\text { Optionald } c & =\text { OneOf }(\text { Mapped singleton } c)(\text { Success }<>), \\
\text { ManyOfD } c s & =\text { Mapped flatten }(\text { Allof }<\text { Optional } c \mid x \in c s>)
\end{aligned}
$$

and

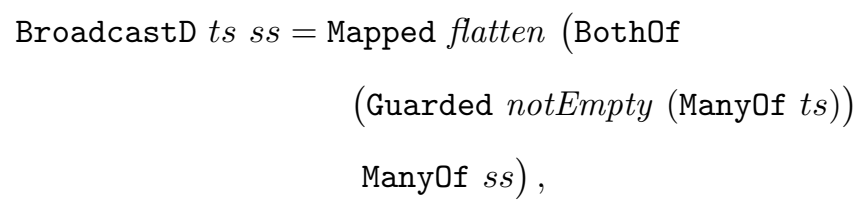

where singleton wraps its parameter value into a singleton list, flatten transforms a structured tuple of lists into one flat list and notEmpty checks whether a list is empty or not. We omit the definitions of these functions.

Notice that this encoding does not use any priority combinators, relying on the the guard to ensure that at least one trigger is present in every allowed interaction.

In order to obtain a similar encoding of a broadcast with maximal progress, one has to tag the lists produced by ManyOfD with their lengths and replace Guarded notEmpty by Maximal $c m p_{2}$ similarly to the previous example.

\subsection{Dynamic Combinators}

Finally, we introduce a set of combinators that allow dynamicity, i.e. creation and deletion of atoms at run time and dynamic reconfiguration of the set of possible interactions among the atoms. Since the connector of a system is static, such dynamicity cannot be realised with the combinators introduced so far and requires additional ones. 


\subsubsection{Dynamic}

Given a port $p$, the Dynamic combinator, binds all existing atoms to the port $p$. Atoms that are created at run time are also bound to the port. This combinator can be thought of as Bind with an atom chosen non-deterministically. Hence, the corresponding type inference rule and semantics are the following:

$$
\begin{gathered}
\frac{(\text { Dynamic } p): \operatorname{sendType}(p) \uparrow \downarrow \text { receiveType }(p)}{} \\
{[\text { Dynamic } p](S)=\{(S(a, p),\{v \mapsto\{(a, p) \mapsto v\} \mid v: \operatorname{receiveType}(p)\}) \mid(a, p) \in \operatorname{domain}(S)\} .}
\end{gathered}
$$

\subsubsection{Joined}

This last combinator that we introduce allows dynamic connector reconfiguration by accepting connectors as values. The connector Joined $c$ acts as a placeholder for connectors passed as upward values by the connector $c$. The type of this connector is thus given by the inference rule

$$
\frac{c:(u \uparrow \downarrow d) \uparrow \downarrow d}{(\text { Joined } c): u \uparrow \downarrow d} .
$$

The connector is named after the natural transformation $\mu$ of monads, called join or multiply, in the context of category theory and join in Haskell. The corresponding semantic function is defined by

$$
\begin{aligned}
& \text { [Joined } c](S)=\left\{\left(x, f_{1} \cup f_{2}\right) \mid\left(c_{1}, f_{1}\right) \in[c](S),\left(x, f_{2}\right) \in\left[c_{1}\right](S)\right. \text { and } \\
& \left.\qquad f_{1} \text { and } f_{2} \text { are downward compatible }\right\} .
\end{aligned}
$$

This connector is very expressive and can be used to derive some of the combinators that we have introduced previously, such as Guarded and Both0f.

\subsubsection{Examples}

Example 3.9. The Joined combinator allows us to redefine Guarded and Both0f as derived combinators:

$$
\begin{aligned}
& \text { Guarded } p c=\text { Joined (Mapped }(\lambda x \text {. if } p(x) \text { then Success } x \text { else Failure }) c), \\
& \text { Both0f } \left.c_{1} c_{2}=\text { Joined (Mapped }\left(\lambda x_{1} \text {. Mapped }\left(\lambda x_{2} \cdot\left(x_{1}, x_{2}\right)\right) c_{2}\right) c_{1}\right) .
\end{aligned}
$$

In both cases, the basic idea is to build a connector from the upward value provided by the underlying connectors using Mapped, then using Joined on this connector. 


\subsection{Closed Semantic Function}

Based on the semantic function we have defined throughout this section, we now introduce the partial function $\llbracket \cdot \rrbracket: \mathcal{C} \rightarrow \mathcal{S} \rightarrow 2^{\mathcal{R}}$ that associates to a connector and a system state, the set of possible assignments defined by the interactions allowed by the connector (see $(2)$ for the definition of close):

$$
\llbracket c \rrbracket(S) \stackrel{d_{e} f}{=}\{\operatorname{close}(o) \mid o \in[c](S)\}, \text { if } c: a \uparrow \downarrow a \text { for some type } a .
$$

This function gives to a connector its meaning as a function from system states to possible assignments.

The following two lemmata provide basic soundness results that we will use to prove the subsequent propositions that show that the closed semantics of any well-typed connector is welldefined and the resulting behaviour satisfies the BIP consistency constraints.

Lemma 3.10. For any connector $c: u \uparrow \downarrow d$ and any system state $S \in \mathcal{S}$, we have $x \in \operatorname{sem}(u)$, for all $(x, f) \in[c](S)$.

Lemma 3.11. For any connector $c: u \uparrow \downarrow d$ and any system state $S \in \mathcal{S}$, we have $\operatorname{domain}(f)=$ $\operatorname{sem}(d)$, for all $(x, f) \in[c](S)$.

The proofs of the lemmata are straightforward by structural induction and are removed into the appendix of the paper.

Proposition 3.12. For any type $u$, the function $\llbracket \cdot \rrbracket$ is well-defined on connectors of type $u \uparrow \downarrow u$. Proof. For any system state $S \in \mathcal{S}$, we have:

$$
\llbracket c \rrbracket(S)=\{\operatorname{close}(o) \mid o \in[c](S)\}=\{f(x) \mid(x, f) \in[c](S)\} .
$$

Consider some $(x, f) \in[c](S)$. By Lemma 3.10, we have $x \in \operatorname{sem}(u)$, whereas, by Lemma 3.11. $\operatorname{sem}(u)=\operatorname{domain}(f)$. If follows immediately that $f(x)$ is well-defined. Hence $\operatorname{close}(\cdot)$ and $\llbracket \cdot \rrbracket$ are also well-defined.

Proposition 3.13. For any type $u$, connector $c \in \mathcal{C}$ of type $u \uparrow \downarrow u$, system state $S \in \mathcal{S}$ and assignment $R \in \llbracket c \rrbracket(S)$, holds the inclusion $\operatorname{domain}(R) \subseteq \operatorname{domain}(S)$.

Proof sketch. This proposition is a straightforward consequence of the construction of the semantics function. It follows directly from the fact that new points can be added to the domain of an assignment only through the Bind and Dynamic combinators. Indeed, all other combinators either leave the assignments untouched or simply combine them. In the Bind and Dynamic combinators, a new atom-port pair is only added to the domain of the assignment if it belongs to the domain of the system state.

Thus, when an atom-port pair $(a, p)$ is part of the domain of an assignment $R \in \llbracket c \rrbracket(S)$, it must be, by construction, the case that $(a, p) \in \operatorname{domain}(S)$. 
Proposition 3.13 means that assignments resulting from closing the connector only send values to atoms through the ports that these atoms are waiting on.

Proposition 3.14. For any type $u$, connector $c \in \mathcal{C}$ of type $u \uparrow \downarrow u$ and system state $S \in \mathcal{S}$, all assignments in $\llbracket c \rrbracket(S)$ involve each of the atoms at most once.

Proof sketch. The proof is direct by structural induction on the connector combinators. The only two cases where special care must be taken, in order to ensure that the assignment does not involve an atom more than once, are Both0f and Joined. In those two cases, the downward compatibility check ensures that this property is not violated.

Notice that the closed semantics of connectors allows simulating the Feedback combinator. This can be achieved by modifying all the combinators higher in the connector tree hierarchy to propagate the corresponding value upward then downward back to the connector, to which Feedback has to be applied. Therefore, the Feedback combinator is not, strictly speaking primitive. However, it greatly simplifies this construction, by allowing feeding values back locally.

\section{Algebraic \& Categorical Properties}

Algebraic and categorical structures often form powerful abstractions in functional programming languages. Such structures, such as monoids, functors and monads [12, as well as many others [13], have been successfully used in the context of functional programming.

In Haskell, those structures form typeclasses such as Monoid, Functor and Monad and can be used very effectively to build high-level expressions. The Monad typeclass is so prevalent that syntactic sugar (the do notation) is present in the language to make its use easier.

In Scala, those structures are not explicitly present. However, many methods, such as map and flatMap mirror the functor and monad operations. Syntactic sugar (the for-notation) is present in the language and, as in Haskell, makes the use of multiple map and flatMap operations syntactically lighter.

In this section, we show that connector combinators form such structures. This allows connectors to be made instances of the corresponding typeclasses and allows programmers to use well-known abstractions and syntactic sugar to build connectors.

\subsection{Algebraic Properties}

Below, we provide two lemmata that show that connector combinators form two monoidal structures. However, first we have to define an equality relation on combinators. This is achieved by a canonical lifting of value and function equalities: 
Definition 4.1 (Connector equality). Two connectors $c_{1}, c_{2} \in \mathcal{C}$ are equal if and only if they define the same open interactions, for any system state, i.e.

$$
c_{1}=c_{2} \stackrel{\text { def }}{\Longleftrightarrow} \forall S \in \mathcal{S},\left[c_{1}\right](S)=\left[c_{2}\right](S)
$$

Lemma 4.2. Connector equality is a congruence w.r.t. all connector combinators introduced in Sect. 3.

Proof sketch. The statement of the lemma follows straightforwardly from the observation that, for a given state $S$, the open-interaction semantics, $[\cdot](S)$, of every combinator involving a sub-connector $c$-i.e. every combinator other than Bind, Success, Failure and Dynamic - is defined per element of $[c](S)$ and without relying on the structure of $c$.

Lemma 4.3. (C , OneOf, Failure) is a commutative monoid, that is OneOf is associative and commutative, with the identity element Failure.

Proof sketch. The lemma trivially follows from the properties of set-theoretical operations.

Lemma 4.4. Given a commutative monoid $(\mathcal{V}, \times, 1)$, the structure $(\mathcal{C}$, Mapped $(\cdot \times \cdot)$ BothOf, Success 1) is also a commutative monoid, that is Mapped $(\cdot \times \cdot)$ Both0f is associative and commutative, with the identity element Success 1.

Proof sketch. The lemma follows directly from the properties of set-theoretical operations and the fact that $(\mathcal{V}, \times, 1)$ is a commutative monoid.

Notice that, taking $\times$ to be the usual Cartesian product, the resulting structure $(\mathcal{C}$, Both0f, Success 1) is a commutative monoid up to isomorphism.

\subsection{Categorical Properties}

Notice that the values $\mathcal{V}$ (comprising data values, functions and connectors) and types introduced in Sect. 2 form a category, whose objects are types, whereas arrows between objects $a$ and $b$ are functions $f: a \rightarrow b \in \mathcal{V}$. We denote this category $T$.

Given types $u$ and $d$, we define two mappings $F_{u p}^{d}, F_{d o w n}^{u}: T \rightarrow T$ by putting, for each type $a$ and each function $f: a \rightarrow b$,

$$
\begin{array}{rlrl}
F_{\text {up }}^{d}(a) & =a \uparrow \downarrow d, & F_{\text {up }}^{d}(f) & =(\text { Mapped } f):(a \uparrow \downarrow d) \\
F_{\text {down }}^{u}(d) & =u \uparrow \downarrow d, \quad F_{\text {down }}^{u}(f) & =(\text { ContraMapped } f): u \uparrow \downarrow b \rightarrow u \uparrow \downarrow a .
\end{array}
$$

The following propositions establish the basic properties of of these mappings, showing that $F_{u p}^{d}$ is a functor-symmetrically, $F_{\text {down }}^{u}$ is a contravariant functor-and that $F_{u p}^{d}$ along with Success and Joined form a monad. The proofs are straightforward and are provided in the appendix. 
Proposition 4.5. For any type $d$, the mapping $F_{u p}^{d}$ is a functor from the category $T$ to itself, that is

$$
\begin{array}{ll}
{\text { Mapped } \text { identity }_{a}} & =\text { identity }_{(a \uparrow \downarrow d)}, \\
\text { Mapped }(g \circ f) & =\text { Mapped } g \circ \text { Mapped } f .
\end{array}
$$

Symmetrically, for any type $u$, the mapping $F_{d o w n}^{u}$ is a contravariant functor from the category $T$ to itself, that is

$$
\begin{array}{ll}
{\text { ContraMapped } \text { identity }_{a}}=\text { identity }_{(a \uparrow \downarrow d)}, \\
\text { ContraMapped }(g \circ f) & =\text { ContraMapped } f \circ \text { ContraMapped } g .
\end{array}
$$

Proposition 4.6. For any type $d$, the functor $F_{u p}^{d}$ along with Success and Joined form a monad, i.e. the following four properties hold:

- Success is a natural transformation from $1_{T}$ to $F_{u p}^{d}$ :

$$
\text { Success } \circ f=\text { Mapped } f \circ \text { Success }
$$

- Joined is a natural transformation from $F_{u p}^{d} \circ F_{u p}^{d}$ to $F_{u p}^{d}$ :

$$
\text { Joined } \circ \text { Mapped (Mapped } f)=\text { Mapped } f \circ \text { Joined, }
$$

- First monad law:

$$
\text { Joined } \circ \text { Mapped Joined }=\text { Joined } \circ \text { Joined }
$$

- Second monad law:

$$
\text { Joined } \circ \text { Mapped Success }=\text { Joined } \circ \text { Success }=i d e n t i t y .
$$

\section{Implementation}

We have implemented the concepts presented in this paper in two functional programming languages, Haskel ${ }^{4}$ and Scald ${ }^{5}$. These implementations allow programmers to build concurrent systems following the principles of BIP in very expressive high-level languages. Thus programmers can separately describe the behaviour and coordination of their system.

Both implementations allow programmers to describe concurrent systems with a set of instructions, which are used to declare the atoms and ports of the system. Special instructions, such as await and spawn are provided for atoms to respectively wait on ports and spawn children atoms. Note that the automata and their description are not directly present in the frameworks. Instead,

${ }^{4}$ https://github.com/redelmann/bip-in-haskell

5 https://github.com/redelmann/bip-in-scala 
data Connector s d u

(a) Connector type in Haskell class Connector $[-\mathrm{D},+\mathrm{U}]$

(b) Connector type in Scala

Figure 12: The Connector type in Haskell and Scala

the behaviour of atoms is expressed directly in the host language, in a fashion that is opaque to the execution engine. The only actions that can be observed by the engine are calls to special instructions such as await and spawn.

Connectors are described using functions and methods which correspond directly to the connector combinators we have introduced in Sect. 3 A library of derived combinators is also made available in both languages. The connector type indicates the types of upward and downward values propagated by the connector. In both cases, connectors are encoded as Generalised Algebraic Data Types (GADTs) as illustrated in Fig. 12 Notice that the Haskell connector type has a type parameter s. This parameter is a phantom type, used to ensure that identifiers do not escape the scope of the system in which they are defined.

As shown in Sect. 4, connectors follow the laws of many interesting algebraic structures. This allows us to make connectors instances of many Haskell typeclasses, such as Monoid, Functor, ProFunctor, Applicative, Alternative, Monad and MonadPlus. These typeclasses allow programmers to build connectors using concepts and functions they already are familiar with.

In Scala, the Connector class implements common methods such as map, flatMap and filter. These methods often mirror those of Haskell typeclasses.

The execution engine is composed of three distinct parts:

The pool of threads is used to concurrently execute the behaviour of atoms.

The system state records information about waiting atoms. It is encoded as a mapping from atoms and ports to:

- nothing, if the atom is not currently waiting on the port;

- the value sent by the atom on the port, along with the continuation of the atom, if the atom is waiting on the port.

The continuations of atoms are stored to avoid needlessly blocking the execution thread of waiting atoms.

The engine core computes possible interactions from the system state and connector. This computation normally takes place when all atoms are either done with their execution or waiting on ports. In Edelmann's Master thesis [10, we investigate a way to execute some of those interactions earlier, while some atoms are still executing. 
We illustrate the implementations of Functional BIP by a concurrent system composed of a producer and 20 consumers. The producer repeatedly produces values that are then sent on the send port of the system. Consumers repeatedly wait to receive a value on the receive port and then directly consume it. The connector of the system states that the values sent by the producer on the send port may be be transmitted to any atom waiting on the receive port. Figure 13 shows a Haskell and a Scala implementations.

We also present a Haskell implementation of a Token Ring system. In this example, atoms are arranged to form a ring. An integer value is exchanged around this ring by the different atoms of the system. The value is incremented at each step until a given maximal value is reached. This example is presented in Fig. 14

\section{Related Work}

We show that an expressive component framework such as BIP can be defined as a DSL based on general purpose functional programming languages. Writing DSL's in functional languages allows their programmers to use powerful modeling concepts and tools with minimal effort. Similar work can be found in [14, 15. Approaches such as [16] also use combinators to describe coordination, but do so locally at each process. These approaches have no notion of a global coordination object such as the connectors we present.

Dynamicity in BIP has been studied by several authors [17, 18, 19]. In [17, the authors present the Dy-BIP framework that allows dynamic reconfiguration of connectors among the ports of the system. They use history variables to allow sequences of interactions with the same instance of a given component type. Functional BIP can emulate history variables using data. Dymanic combinators allow reconfiguration of connectors, but also creation and deletion of atoms, thereby extending the expressivity with respect to Dy-BIP.

In [19], the authors revisit the BIP expressiveness, by introducing simple behaviour, such as prefixing, in the BIP glue operators. They show that such minor modifications can rapidly lead to Turing completeness of glue. In contrast to [19] and other frameworks, such as [20, 21, Functional BIP relies on a clear distinction between behaviour and coordination expressed by memoryless connectors obtained by combinator composition. From this perspective, the approach in [18] is closest to the one we adopted in Functional BIP. Although, the expressiveness of the two approaches seems very close, we leave the formal investigation for future work.

In contrast to other formalisms such as 22] our framework supports full dynamism and is rooted in rigorous abstract semantics. In [23], dynamic architectures are defined as a set of global transitions between global configurations. These transitions are expressed in a first order logic extended with architecture-specific predicates. The same logic is used in [24, 25] but global configurations are computed at run time from the local constraints of each component. 26] provides an operational 


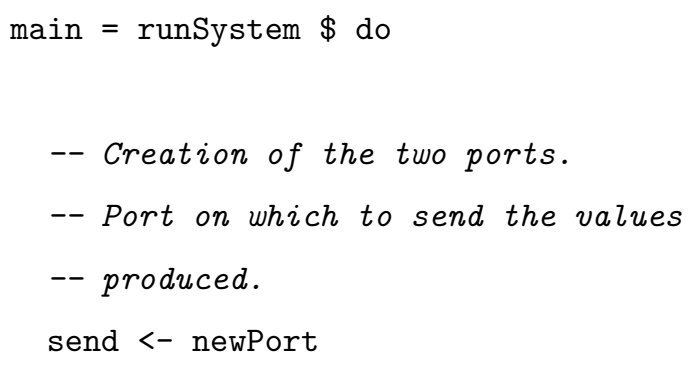

(a) Haskell

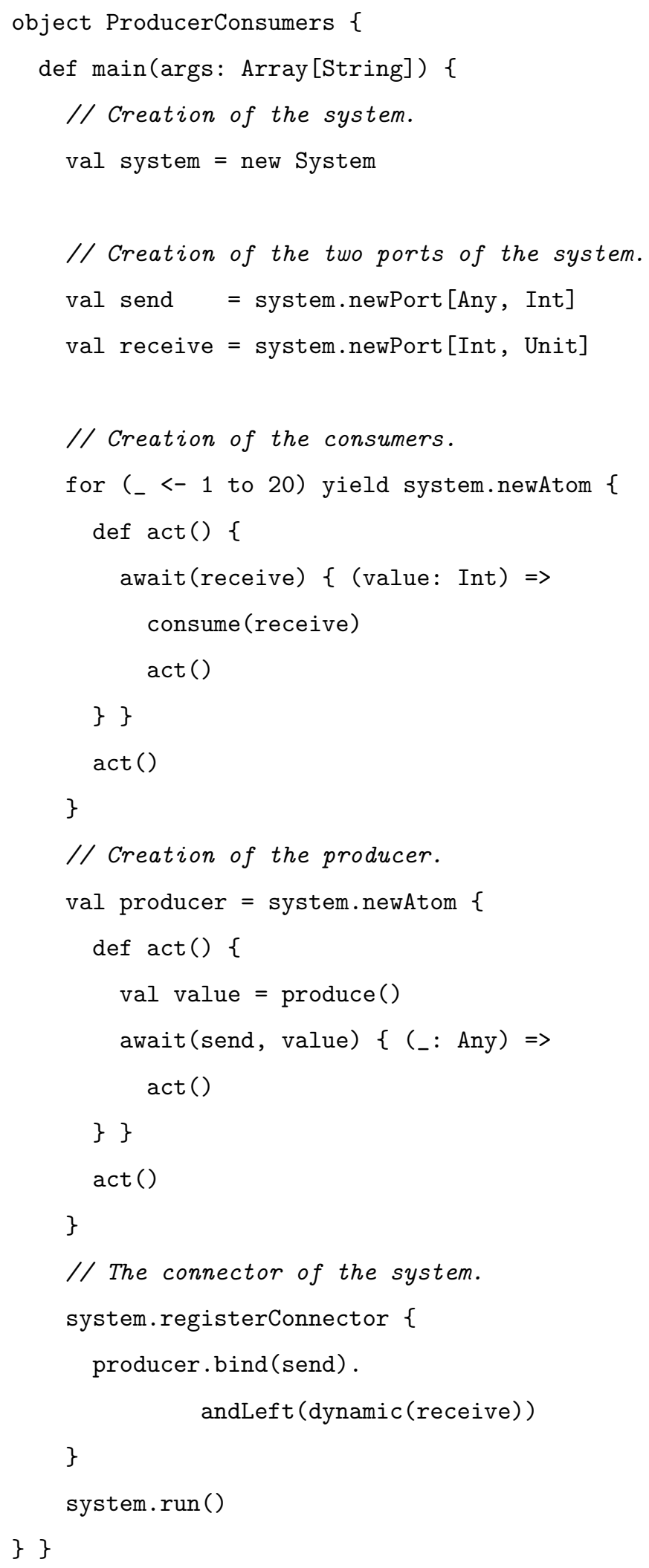

(b) Scala

Figure 13: Producer-Consumers example implemented in Haskell and Scala 


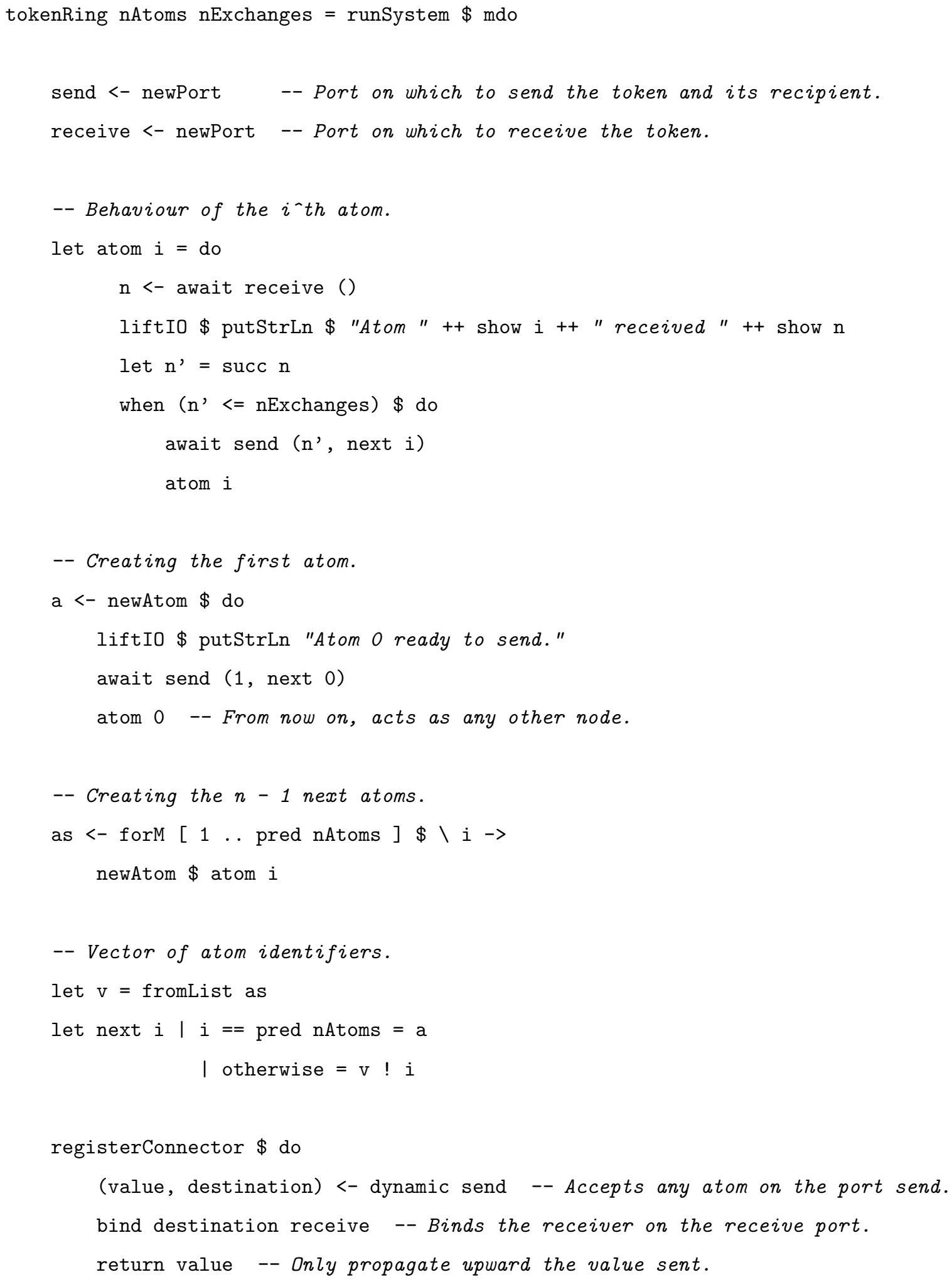

Figure 14: Token Ring example implemented in Haskell 
semantics based on the composition of global configurations from local ones. These express three forms of dependencies between services (mandatory, optional and negative). Nonetheless, dynamism is supported only at the installation phase.

\section{Conclusion}

The paper shows how the BIP component framework can be embedded in functional host languages. The embedding consists in defining sets of connector combinators that can describe the coordination mechanisms of BIP. The definition is progressive and incremental. We first define combinators to express synchronisation and associated data transfer between the components of a system. Then, we have introduced combinators for the application of priority policies allowing conflict resolution between enabled interactions. Finally, we have presented combinators to deal with the dynamic creation of components and connectors.

We have shown that the set of the defined combinators enjoy interesting algebraic properties and

form well-known algebraic structures which are of particular importance for the implementation of connectors in functional programming languages.

The two implementations show how these concepts can be transposed, respectively, into Haskell and Scala. For both languages, we have released an open source framework which programmers can use to build concurrent systems using the high-level coordination primitives offered by BIP. 


\section{References}

[1] A. Basu, S. Bensalem, M. Bozga, J. Combaz, M. Jaber, T.-H. Nguyen, J. Sifakis, Rigorous component-based system design using the BIP framework, IEEE Software 28 (3) (2011) 41-48.

[2] S. Bliudze, J. Sifakis, The algebra of connectors - structuring interaction in BIP, IEEE Transactions on Computers 57 (10) (2008) 1315-1330.

[3] E. Baranov, S. Bliudze, A note on the expressiveness of BIP in: Proceedings Combined 23rd International Workshop on Expressiveness in Concurrency and 13th Workshop on Structural Operational Semantics (EXPRESS/SOS 2016), Vol. 222 of EPTCS, 2016, pp. 1-14. doi:10.4204/EPTCS.222.1, URL http://dx.doi.org/10.4204/EPTCS.222.1

[4] S. Bliudze, J. Sifakis, A notion of glue expressiveness for component-based systems, in: CONCUR 2008, Springer, 2008, pp. 508-522.

[5] S. Bliudze, A. Mavridou, R. Szymanek, A. Zolotukhina, Coordination of software components with BIP: Application to OSGi, in: Proceedings of the 6th International Workshop on Modeling in Software Engineering, MiSE 2014, ACM, New York, NY, USA, 2014, pp. 25-30. doi:10.1145/2593770.2593777.

[6] S. Bliudze, J. Sifakis, M. D. Bozga, M. Jaber, Architecture internalisation in BIP, in: Proceedings of the 17th International ACM Sigsoft Symposium on Component-based Software Engineering, CBSE '14, ACM, 2014, pp. 169-178. doi:10.1145/2602458.2602477.

[7] S. Bliudze, J. Sifakis, M. D. Bozga, M. Jaber, Architecture internalisation in BIP, in: CBSE '14, ACM, 2014, pp. 169-178.

[8] S. Marlow (Ed.), Haskell 2010 language report, Haskell.org, 2010, https://www.haskell. org/definition/haskell2010.pdf.

[9] M. Odersky, P. Altherr, V. Cremet, B. Emir, S. Maneth, S. Micheloud, N. Mihaylov, M. Schinz, E. Stenman, M. Zenger, An overview of the Scala programming language, Tech. rep., EPFL (2004).

URL http://infoscience.epfl.ch/record/52656

[10] R. Edelmann, Behaviour-Interaction-Priority in functional programming languages: Formalisation and implementation of concurrency frameworks in Haskell and Scala, Master's thesis, Ecole polytechnique fédérale de Lausanne (EPFL) (Jan. 2015).

URL http://infoscience.epfl.ch/record/215767 
[11] R. Edelmann, S. Bliudze, J. Sifakis, Functional BIP: Embedding connectors in functional programming languages, brief announcement at the 9th Interaction and Concurrency Experience workshop (ICE 2016) (Jun. 2016).

[12] P. Wadler, Monads for functional programming, in: Advanced Functional Programming, Springer, 1995, pp. 24-52.

[13] B. Yorgey, The typeclassopedia, The Monad.Reader 13 (2009) 17-68.

[14] P. Haller, M. Odersky, Scala actors: Unifying thread-based and event-based programming, Theoretical Computer Science 410 (2) (2009) 202-220.

[15] J. Launchbury, T. Elliott, Concurrent orchestration in Haskell, SIGPLAN Not. 45 (11) (2010) 79-90. doi:10.1145/2088456.1863534.

URL http://doi .acm.org/10.1145/2088456.1863534

[16] K. Donnelly, M. Fluet, Transactional events, SIGPLAN Not. 41 (9) (2006) 124-135. doi: 10.1145/1160074.1159821

URL http://doi.acm.org/10.1145/1160074.1159821

[17] M. Bozga, M. Jaber, N. Maris, J. Sifakis, Modeling dynamic architectures using Dy-BIP, in: Software Composition, Springer, 2012, pp. 1-16.

[18] R. Bruni, H. C. Melgratti, U. Montanari, Behaviour, interaction and dynamics, in: Specification, Algebra, and Software - Essays Dedicated to Kokichi Futatsugi, Vol. 8373 of LNCS, Springer, 2014, pp. 382-401. doi:10.1007/978-3-642-54624-2_19.

URL http://dx.doi.org/10.1007/978-3-642-54624-2_19

[19] C. Di Giusto, J.-B. Stefani, Revisiting glue expressiveness in component-based systems, in: COORDINATION 2011, Springer, 2011, pp. 16-30. doi:10.1007/978-3-642-21464-6_2.

URL http://dx.doi.org/10.1007/978-3-642-21464-6_2

[20] P. Inverardi, A. L. Wolf, Formal specification and analysis of software architectures using the chemical abstract machine model, Software Engineering, IEEE Transactions on 21 (4) (1995) 373-386.

[21] D. Le Métayer, Describing software architecture styles using graph grammars, Software Engineering, IEEE Transactions on 24 (7) (1998) 521-533.

[22] J. Magee, J. Kramer, Dynamic structure in software architectures, SIGSOFT Softw. Eng. Notes 21 (6) (1996) 3-14. doi:10.1145/250707.239104.

URL http://doi .acm.org/10.1145/250707.239104 
[23] J. S. Kim, D. Garlan, Analyzing architectural styles, Journal of Systems and Software 83 (7) (2010) 1216-1235.

[24] I. Georgiadis, J. Magee, J. Kramer, Self-organising software architectures for distributed systems, in: Self-healing systems, ACM, 2002, pp. 33-38.

[25] M. H. Kacem, M. Jmaiel, A. H. Kacem, K. Drira, Evaluation and comparison of ADL based approaches for the description of dynamic of software architectures, in: ICEIS (3), 2005, pp. $189-195$.

[26] M. Belguidoum, F. Dagnat, Dependency management in software component deployment, Electronic Notes in Theoretical Computer Science 182 (2007) 17-32. 


\section{Appendix A. Additional proofs}

Proof of Lemma 3.10: For any connector $c: u \uparrow \downarrow d$ and any system state $S \in \mathcal{S}$, we have $x \in \operatorname{sem}(u)$, for all $(x, f) \in[c](S)$.

Proof. We prove the lemma by structural induction on the typing rules.

\section{Case Bind $a p$}

Let $c=$ Bind $a p$, with some atom $a \in \mathcal{A}$ and port $p \in P$. Then, by the typing rule of Bind (3) $u=\operatorname{sendType}(p)$ and $d=$ receiveType $(p)$. If $(a, p) \in \operatorname{domain}(S)$, by (4), $x=S(a, p)$.

Furthermore, by the definition of state(1),

$$
S(a, p) \in \operatorname{sem}(\operatorname{sendType}(p))=\operatorname{sem}(u)
$$

If $(a, p) \notin \operatorname{domain}(S)$, then the proposition trivially holds, as, by $(4)$ ] [Bind $a p](S)=\emptyset$.

\section{Case Success}

Let $c=\operatorname{Success} v$, with some value $v \in \mathcal{V}$. Then, by the typing rule of Succes: (5), it must be the case that $v: u$ and thus that $v \in \operatorname{sem}(u)$. By (6), we have $x=v$. Thus the proposition trivially holds.

\section{Case Failure}

Let $c=$ Failure. Then, by $(7)][c](S)=\emptyset$ and the proposition trivially holds.

\section{Case OneOf}

Let $c=$ OneOf $c_{1} c_{2}$. Then, by the typing rule of OneOf (8), it must be the case that $c_{1}: u \uparrow \downarrow d$ and $c_{2}: u \uparrow \downarrow d$. By induction hypothesis, the proposition holds for $c_{1}$ and $c_{2}$. Thus, the proposition holds for $c$, as, by $(9)][c](S)=\left[c_{1}\right](S) \cup\left[c_{2}\right](S)$.

\section{Case BothOf}

Let $c=\operatorname{BothOf} c_{1} c_{2}$. Then, by the typing rule of Both0f (10) it must be the case that:

- $c_{1}: v \uparrow \downarrow d$ for some type $v$,

- $c_{2}: w \uparrow \downarrow d$ for some type $w$,

- $u=v \times w$.

Furthermore, by (11) for any $(x, f) \in[c](S)$, we have $x=\left(x_{1}, x_{2}\right)$ and $f=f_{1} \cup f_{2}$, such that $\left(x_{1}, f_{1}\right) \in\left[c_{1}\right](S)$ and $\left(x_{2}, f_{2}\right) \in\left[c_{2}\right](S)$. By the induction hypothesis, the proposition holds for $c_{1}$ and $c_{2}$, that is $x_{1} \in \operatorname{sem}(v)$ and $x_{2} \in \operatorname{sem}(w)$. Hence, $x=\left(x_{1}, x_{2}\right) \in \operatorname{sem}(u)$.

\section{Case Mapped}

Let $c=$ Mapped $g c_{1}$. Then, by the typing rule of Mapped (20), we have that $c_{1}: v \uparrow \downarrow d$, for 
some type $v$, and $g: v \rightarrow u$. Furthermore, by (21), for any $(x, f) \in[c](S)$, we have $x=g\left(x_{1}\right)$, such that $\left(x_{1}, f_{1}\right) \in\left[c_{1}\right](S)$, for some downward function $f_{1}$. By induction hypothesis, we have $x_{1} \in \operatorname{sem}(v)$ and, consequently, $x=g\left(x_{1}\right) \in \operatorname{sem}(u)$.

\section{Case ContraMapped}

Let $c=$ ContraMapped $g c_{1}$. Then, by the typing rule of ContraMapped (20), it must be the case that $c_{1}: u \uparrow \downarrow e$ for some type $e$. Furthermore, by $(22)$, for any $(x, f) \in[c](S)$, we have $\left(x, f_{1}\right) \in\left[c_{1}\right](S)$, with some downward function $f_{1}$, such that $f=f_{1} \circ g$. Thus $x \in \operatorname{sem}(u)$ by the induction hypothesis.

\section{Case Guarded}

Let $c=$ Guarded $g c_{1}$. Then, by the typing rule of Guarded (23), we have that:

- $g: u \rightarrow$ bool and

- $c_{1}: u \uparrow \downarrow d$.

Furthermore, by (24) for any $(x, f) \in[c](S)$, we have $(x, f) \in\left[c_{1}\right](S)$ and $g(x)=$ true. Thus, $x \in \operatorname{sem}(u)$, by the induction hypothesis.

\section{Case Feedback}

Let $c=$ Feedback $c_{1}$. By the typing rule of Feedback (25), we have $c_{1}: u \uparrow \downarrow(d \times u)$. By $(26)$ for any $(x, f) \in[c](S)$, we have $(x, g) \in\left[c_{1}\right](S)$, with some downward function $g$. Thus, the proposition holds trivially by the induction hypothesis.

\section{Case First0f}

Let $c=$ Firstof $c_{1} c_{2}$. Then, by the typing rule of First0f (16) it must be the case that $c_{1}: u \uparrow \downarrow d$ and $c_{2}: u \uparrow \downarrow d$. Furthermore, by (17), for any $(x, f) \in[c](S)$, we have either $(x, f) \in\left[c_{1}\right](S)$ or $(x, f) \in\left[c_{2}\right](S)$. Thus, the proposition trivially holds by the induction hypothesis.

\section{Case Maximal}

Let $c=$ Maximal $g c_{1}$, then, by the typing rule of Maximal (18) we must have that $c_{1}: u \uparrow \downarrow d$ and $g:(u \times u) \rightarrow$ bool. Furthermore, by $[19)][c](S) \subseteq\left[c_{1}\right](S)$. Thus, for any $(x, f) \in[c](S)$, we also have $(x, f) \in\left[c_{1}\right](S)$ and the proposition holds by the induction hypothesis.

\section{Case Dynamic}

Let $c=$ Dynamic $p$ for some port $p \in P$. By the typing rule of Dynamic(27), we have $u=\operatorname{sendType}(p)$. Furthermore, by $(28)$, for any $(x, f) \in[c](S)$, we have $x=S(a, p)$, for some atom $a$, such that $(a, p) \in \operatorname{domain}(S)$. By the definition of state (see Sect. 2.7), we have $x: \operatorname{sendType}(p)$, hence $x \in \operatorname{sem}(u)$. 


\section{Case Joined}

Let $c=$ Joined $c_{1}$. Then, by the typing rule of Joined (29), it must be the case that $c_{1}:(u \uparrow \downarrow d) \uparrow \downarrow d$. Furthermore, by $(30)$, for any $(x, f) \in[c](S)$, we have $f=f_{1} \cup f_{2}$, with $\left(x, f_{1}\right) \in\left[c_{2}\right](S)$ for some connector $c_{2}: u \uparrow \downarrow d$, such that $\left(c_{2}, f_{2}\right) \in\left[c_{1}\right](S)$. Notice that, by the induction hypothesis, we, indeed, have $c_{2} \in \operatorname{sem}(u \uparrow \downarrow d)$. Hence, by a second application of the induction hypothesis, $x \in \operatorname{sem}(u)$.

Proof of Lemma 3.11: For any connector $c: u \uparrow \downarrow d$ and any system state $S \in \mathcal{S}$, we have $\operatorname{domain}(f)=\operatorname{sem}(d)$, for all $(x, f) \in[c](S)$.

Proof. We prove the lemma by structural induction on the typing rules.

\section{Case Bind $a p$}

Let $c=$ Bind $a p$, for some atom $a \in \mathcal{A}$ and port $p \in P$. Then, by the typing rule of Bind (3), it must be the case that $c: \operatorname{sendType}(p) \uparrow \downarrow$ receiveType $(p)$. Furthermore, by (4), if $(a, p) \in \operatorname{domain}(S)$, then, for any $(x, f) \in[c](S)$, the domain of $f$ is sem(receiveType $(p))$, i.e. $\operatorname{domain}(f)=\operatorname{sem}(d)$. If $(a, p) \notin \operatorname{domain}(S)$, then the proposition trivially holds, as $[$ Bind $a p](S)=\emptyset$.

\section{Case Success}

Let $c=\operatorname{Success} v$, with some value $v: u$. Then, by $(6)$, for any $(x, f) \in[c](S)$ we have $\operatorname{domain}(f)=\operatorname{sem}(d)$. Thus, the proposition trivially holds.

\section{Case Failure}

Let $c=$ Failure. Then, by $(7)][c](S)=\emptyset$ and the proposition trivially holds.

\section{Case OneOf}

Let $c=$ OneOf $c_{1} c_{2}$. Then, by the typing rule of OneOf (8) it must be the case that $c_{1}: u \uparrow \downarrow d$ and $c_{2}: u \uparrow \downarrow d$. Furthermore, by $(9)$, for any $(x, f) \in[c](S)$, we either have $(x, f) \in\left[c_{1}\right](S)$ or $(x, f) \in\left[c_{2}\right](S)$. In both cases, we have $\operatorname{domain}(f)=\operatorname{sem}(d)$ by the induction hypothesis.

\section{Case Both0f}

Let $c=\operatorname{Both0f} c_{1} c_{2}$. Then, by the typing rule of Bothof (10) it must be the case that:

- $c_{1}: v \uparrow \downarrow d$ for some type $v$.

- $c_{2}: w \uparrow \downarrow d$ for some type $w$.

Furthermore, by (11), for any $(x, f) \in[c](S)$, we have $x=\left(x_{1}, x_{2}\right)$ and $f=f_{1} \cup f_{2}$, for some $\left(x_{1}, f_{1}\right) \in\left[c_{1}\right](S)$ and $\left(x_{2}, f_{2}\right) \in\left[c_{2}\right](S)$. By the induction hypothesis, domain $\left(f_{1}\right)=$ $\operatorname{domain}\left(f_{2}\right)=\operatorname{sem}(d)$. Hence, also $\operatorname{domain}(f)=\operatorname{sem}(d)$. 


\section{Case Mapped}

Let $c=$ Mapped $g c_{1}$. Then, by the typing rule for Mapped (20), it must be the case that $c_{1}: v \uparrow \downarrow d$ and $g: v \rightarrow u$. Furthermore, by $(21)$ for any $(x, f) \in[c](S)$, we have $(x, f)=(g(y), f)$, for some $(y, f) \in\left[c_{1}\right](S)$ and the propostion holds, by the induction hypothesis.

\section{Case ContraMapped}

Let $c=$ ContraMapped $g c_{1}$, then by the typing rule of ContraMapped (20), we have that $c_{1}: u \uparrow \downarrow e$, for some type $e$, and $g: d \rightarrow e$, i.e. $\operatorname{domain}(g)=\operatorname{sem}(d)$. Furthermore, for any $(x, f) \in[c](S)$, by $(22)$, we have $(x, f)=\left(x, f_{1} \circ g\right)$, for some $\left(x, f_{1}\right) \in\left[c_{1}\right](S)$.

By the induction hypothesis, we have $\operatorname{sem}(e)=\operatorname{domain}\left(f_{1}\right)$. Thus, as $\operatorname{range}(g) \subseteq \operatorname{sem}(e)=$ $\operatorname{domain}\left(f_{1}\right)$, the composition $f_{1} \circ g$ is well-defined and we have $\operatorname{domain}\left(f_{1} \circ g\right)=\operatorname{domain}(g)=$ $\operatorname{sem}(d)$.

\section{Case Guarded}

Let $c=$ Guarded $g c_{1}$. Then, by the typing rule of Guarded (23), we have that:

- $g: u \rightarrow$ bool and

- $c_{1}: u \uparrow \downarrow d$.

Furthermore, by (24)] for any $(x, f) \in[c](S)$, we have $(x, f) \in\left[c_{1}\right](S)$ and $g(x)=$ true. Thus, $\operatorname{domain}(f)=\operatorname{sem}(d)$, by the induction hypothesis.

\section{Case Feedback}

Let $c=$ Feedback $c_{1}$. Then, by the typing rule of Feedback (25), it must be the case that $c_{1}: u \uparrow \downarrow(d \times u)$. Furthermore, for any $(x, f) \in[c](S)$, by $(26)$ we have $(x, f)=\left(x, g \circ \operatorname{tag}_{x}\right)$, for some $(x, g) \in\left[c_{1}\right](S)$, with $\operatorname{tag}_{x}(y) \stackrel{\text { def }}{=}(y, x)$.

By Lemma 3.10, we have $x \in \operatorname{sem}(u)$. Hence, $\operatorname{tag}_{x}: d \rightarrow(d, u)$, i.e. $\operatorname{range}\left(\operatorname{tag}_{x}\right) \subseteq \operatorname{sem}(d \times u)$. By the induction hypothesis, we have $\operatorname{sem}(d \times u)=\operatorname{domain}(g)$. Hence, the composition $g \circ \operatorname{tag}_{x}$ is well-defined and $\operatorname{domain}(f)=\operatorname{domain}\left(\operatorname{tag}_{x}\right)=\operatorname{sem}(d)$.

\section{Case Firstof}

Let $c=$ Firstof $c_{1} c_{2}$. Then, by the typing rule of First0f (16)] it must be the case that $c_{1}: u \uparrow \downarrow d$ and $c_{2}: u \uparrow \downarrow d$. Furthermore, by (17), for any $(x, f) \in[c](S)$, we have either $(x, f) \in\left[c_{1}\right](S)$ or $(x, f) \in\left[c_{2}\right](S)$. Thus, the proposition trivially holds by the induction hypothesis.

\section{Case Maximal}

Let $c=$ Maximal $g c_{1}$, then, by the typing rule of Maximal[(18) we must have that $c_{1}: u \uparrow \downarrow d$ 
and $g:(u \times u) \rightarrow$ bool. Furthermore, by $(19)][c](S) \subseteq\left[c_{1}\right](S)$. Thus, for any $(x, f) \in[c](S)$, we also have $(x, f) \in\left[c_{1}\right](S)$ and the proposition holds by the induction hypothesis.

\section{Case Dynamic $p$}

Let $c=$ Dynamic $p$, with some port $p \in P$. Then, by the typing rule for Dynamic(27), we have $d=$ receive Type $(p)$. Furthermore, by $(28)$, for any $(x, f) \in[c](S)$, we have domain $(f)=$ $\operatorname{sem}($ receiveType $(p))$. Thus, the proposition trivially holds.

Case Joined

Let $c=$ Joined $c_{1}$. Then, by the typing rule of Joined (29), it must be the case that $c_{1}:(u \uparrow \downarrow d) \uparrow \downarrow d$. Furthermore, by $(30)$, for any $(x, f) \in[c](S)$, we have $f=f_{1} \cup f_{2}$, with $\left(x, f_{1}\right) \in\left[c_{2}\right](S)$ for some connector $c_{2}$, such that $\left(c_{2}, f_{2}\right) \in\left[c_{1}\right](S)$. Hence, $\operatorname{domain}(f)=$ $\operatorname{domain}\left(f_{1}\right)=\operatorname{domain}\left(f_{2}\right)$.

By Lemma 3.10, $c_{2} \in \operatorname{sem}(u \uparrow \downarrow d)$, that is, indeed, $c_{2}: u \uparrow \downarrow d$. Hence, by the induction hypothesis, $\operatorname{domain}\left(f_{1}\right)=\operatorname{domain}\left(f_{2}\right)=\operatorname{sem}(d)$. Hence, domain $(f)=\operatorname{domain}\left(f_{1}\right)=$ $\operatorname{domain}\left(f_{2}\right)=\operatorname{sem}(d)$.

Proof of Proposition 4.5: For any type d, the mappings $F_{u p}^{d}$ and $F_{d o w n}^{u}$ are, respectively, covariant and contravariant functors from the category $T$ to itself.

We will only prove the proposition statement for $F_{u p}^{d}$. The statement for $F_{d o w n}^{u}$ is symmetrical. Proof. To show that $F_{u p}^{d}$ is a functor, we show that identity and composition are preserved.

Identity We have to prove that, for any two types $u$ and $d, F_{u p}^{d}\left(1_{u}\right)=1_{F_{u p}^{d}(u)}$. Let $u$ by any type. By definition, $1_{u}$ is the identity function identity $: u \rightarrow u$. Therefore, we have that:

$$
F_{u p}^{d}\left(1_{u}\right)=\text { Mapped identity }{ }_{u}
$$

We observe that $1_{F_{u p}^{d}(u)}$ is the identity function that maps connectors of type $u \uparrow \downarrow d$ to themselves. Therefore, for the identity preservation property to hold, we must have that for any connector $c$ of the correct type the following holds:

$$
\text { Mapped identity } c=c \text {. }
$$

By definition, the above equality is equivalent to the following statement:

$$
\forall S \in \mathcal{S} .[\text { Mapped identity } c](S)=[c](S)
$$


Let $S$ be any system state. We have that the above statement is trivially true as:

$$
\begin{aligned}
\text { Mapped identity } \left._{u} c\right](S) & =\left\{\left(\text { identity }_{u}(x), f\right) \mid(x, f) \in[c](S)\right\} \\
& =\{(x, f) \mid(x, f) \in[c](S)\} \\
& =[c](S) .
\end{aligned}
$$

Composition We are left to show that indeed $F_{u p}^{d}(g \circ h)=F_{u p}^{d}(g) \circ F_{u p}^{d}(h)$, i.e. that

$$
\text { Mapped }(g \circ h)=(\text { Mapped } g) \circ(\text { Mapped } h),
$$

that is, for any connector $c$ of appropriate type, we must have that:

$$
\text { Mapped }(g \circ h) c=\text { Mapped } g(\text { Mapped } h c) \text {. }
$$

By Definition 4.1, this equality reduces to:

$$
\forall S \in \mathcal{S} .[\text { Mapped }(g \circ h) c](S)=[\text { Mapped } g(\text { Mapped } h c)](S)
$$

Let $S$ be any system state. We trivially have that:

$$
\begin{aligned}
\text { [Mapped }(g \circ h) c](S) & =\{(g(h(x)), h) \mid(x, h) \in[c](S)\} \\
& =\{(g(y), h) \mid(y, h) \in[\text { Mapped } h c](S)\} \\
& =[\text { Mapped } g \text { (Mapped } h c)](S) .
\end{aligned}
$$

Proof of Proposition 4.6: For any type d, the functor $F_{u p}^{d}: T \rightarrow T$ along with Success and Joined form a monad.

In order to prove the proposition, we consider two families of mappings $\eta: 1_{T} \rightarrow F_{u p}^{d}$ and $\mu: F_{u p}^{d} \circ F_{u p}^{d} \rightarrow F_{u p}^{d}$, defined as follows (recall that the objects of $T$ are types):

- for each type $u$, the mapping $\eta_{u}: u \rightarrow F_{u p}^{d}(u)$ associates, to each $v \in \operatorname{sem}(u)$, the connector (Success $v): u \uparrow \downarrow d$;

- for each type $u$, the mapping $\mu_{u}:(u \uparrow \downarrow d) \uparrow \downarrow d \rightarrow u \uparrow \downarrow d$ associates, to each $c:(u \uparrow \downarrow d) \uparrow \downarrow d$, the connector (Joined $c$ ) : $u \uparrow \downarrow d$.

We will show that both these families are natural transformations and that, together with $F_{u p}^{d}$, they satisfy the necessary coherence conditions.

\section{Proof.}


$\eta: 1_{T} \rightarrow F_{u p}^{d}$ is a natural transformation. We have to prove that the following equality holds for any function $g: a \rightarrow b$ :

$$
\left(\eta_{b} \circ 1_{T}\right)(g)=F_{u p}^{d}(g) \circ \eta_{a}
$$

Which, in this context, translates to:

$$
\text { Success } \circ g=(\text { Mapped } g) \circ \text { Success }
$$

Or, for any value $v \in \operatorname{sem}(a)$ :

$$
\text { Success } g(v)=\text { Mapped } g(\operatorname{Success} v)
$$

Which is trivially true by definition of the semantics.

$\mu: F_{u p}^{d} \circ F_{u p}^{d} \rightarrow F_{u p}^{d}$ is a natural transformation. We have to prove that the following equality holds, for any function $g: a \rightarrow b$,

$$
\left(\mu_{b} \circ\left(F_{u p}^{d} \circ F_{u p}^{d}\right)\right)(g)=F_{u p}(g) \circ \mu_{a} .
$$

Which, in this context, translates to

$$
\text { Joined } \circ \text { Mapped }(\text { Mapped } g)=(\text { Mapped } g) \circ \text { Joined }
$$

or, for any connector $c:(u \uparrow \downarrow d) \uparrow \downarrow d$,

$$
\text { Joined }(\text { Mapped }(\text { Mapped } g) c)=\text { Mapped } g(\text { Joined } c) \text {. }
$$

By definition of the equality on connectors, this is equivalent to the following statement:

$$
\forall S \in \mathcal{S} \text {.[Joined (Mapped }(\text { Mapped } g) c)](S)=[\text { Mapped } g(\text { Joined } c)](S)
$$

Let $S$ be any system state. We have that:

$$
\begin{aligned}
& \text { [Joined (Mapped (Mapped } g) c)](S) \\
& \begin{array}{c}
=\left\{\left(x, f_{1} \cup f_{2}\right) \mid\left(c_{1}, f_{1}\right) \in[\text { Mapped (Mapped } g) c\right](S),\left(x, f_{2}\right) \in\left[c_{1}\right](S) \text { and } \\
\left.\qquad f_{1} \text { and } f_{2} \text { are downward compatible }\right\}
\end{array} \\
& =\left\{\left(x, f_{1} \cup f_{2}\right) \mid\left(c_{1}, f_{1}\right) \in[c](S),\left(x, f_{2}\right) \in\left[\text { Mapped } g c_{1}\right](S)\right. \text { and } \\
& \left.\qquad f_{1} \text { and } f_{2} \text { are downward compatible }\right\} \\
& =\left\{\left(g(x), f_{1} \cup f_{2}\right) \mid\left(c_{1}, f_{1}\right) \in[c](S),\left(x, f_{2}\right) \in\left[c_{1}\right](S)\right. \text { and } \\
& \left.f_{1} \text { and } f_{2} \text { are downward compatible }\right\} \\
& =[\text { Mapped } g(\text { Joined } c)](S) .
\end{aligned}
$$


Identity. We have to show that, for any type $u$ and any connector $c: u \uparrow \downarrow v$,

$$
\left(\mu_{u} \circ F_{u p}^{d}\left(\eta_{u}\right)\right)(c)=\left(\mu_{u} \circ \eta_{F_{u p}^{d}(u)}\right)(c)=c,
$$

which translates to:

$$
\text { Joined }(\text { Mapped Success } c)=\text { Joined }(\operatorname{Success} c)=c \text {. }
$$

For any system state $S$, we have

$$
\begin{aligned}
& \text { [Joined (Mapped Success } c)](S) \\
& =\left\{\left(x, f_{1} \cup f_{2}\right) \mid\left(c_{1}, f_{1}\right) \in[\text { Mapped Success } c](S),\left(x, f_{2}\right) \in\left[c_{1}\right](S)\right. \text { and } \\
& f_{1} \text { and } f_{2} \text { are downward compatible\} } \\
& =\left\{\left(x, f_{1} \cup f_{2}\right) \mid\left(c_{1}, f_{1}\right) \in[c](S),\left(x, f_{2}\right) \in\left[\text { Success } c_{1}\right](S)\right. \text { and } \\
& \left.f_{1} \text { and } f_{2} \text { are downward compatible }\right\} \\
& =\left\{\left(c_{1}, f_{1}\right) \mid\left(c_{1}, f_{1}\right) \in[c](S)\right\} \\
& =[c](S) \\
& \text { [Joined }(\text { Success } c)](S) \\
& =\left\{\left(x, f_{1} \cup f_{2}\right) \mid\left(c_{1}, f_{1}\right) \in[\operatorname{Success} c](S),\left(x, f_{2}\right) \in\left[c_{1}\right](S)\right. \text { and } \\
& f_{1} \text { and } f_{2} \text { are downward compatible } \\
& =\left\{\left(x, f_{2}\right) \mid\left(x, f_{2}\right) \in[c](S)\right\} \\
& =[c](S) \text {. }
\end{aligned}
$$

Associativity. We have to show that, for any type $u$ and any connector $c:((u \uparrow \downarrow d) \uparrow \downarrow d) \uparrow \downarrow d$,

$$
\left(\mu_{u} \circ F_{u p}^{d}\left(\mu_{u}\right)\right)(c)=\left(\mu_{u} \circ \mu_{F_{u p}^{d}(u)}\right)(c)
$$

which translates to The first law translates in this context to:

$$
\text { Joined }(\text { Mapped Joined } c)=\text { Joined }(\text { Joined } c)
$$


For any system state $S$, we have

$$
\begin{aligned}
& \text { [Joined (Mapped Joined } c)](S) \\
& \qquad \begin{array}{c}
=\left\{\left(x, f_{1} \cup f_{2}\right) \mid\left(c_{1}, f_{1}\right) \in[\text { Mapped Joined } c](S),\left(x, f_{2}\right) \in\left[c_{1}\right](S)\right. \text { and } \\
\left.\qquad f_{1} \text { and } f_{2} \text { are downward compatible }\right\}
\end{array} \\
& =\left\{\left(x, f_{1} \cup f_{2}\right) \mid\left(c_{1}, f_{1}\right) \in[c](S),\left(x, f_{2}\right) \in\left[\text { Joined } c_{1}\right](S)\right. \text { and } \\
& \left.\qquad f_{1} \text { and } f_{2} \text { are downward compatible }\right\} \\
& =\left\{\left(x, f_{1} \cup f_{2} \cup f_{3}\right) \mid\left(c_{1}, f_{1}\right) \in[c](S),\left(c_{3}, f_{3}\right) \in\left[c_{1}\right](S),\left(x, f_{2}\right) \in\left[c_{3}\right](S)\right. \text { and } \\
& \left.\qquad f_{1}, f_{2} \text { and } f_{3} \text { are downward compatible }\right\} \\
& =\left\{\left(x, f_{2} \cup f_{3}\right) \mid\left(c_{3}, f_{3}\right) \in[\text { Joined } c](S),\left(x, f_{2}\right) \in\left[c_{3}\right](S)\right. \text { and } \\
& \left.\quad f_{2} \text { and } f_{3} \text { are downward compatible }\right\} \\
& =[\text { Joined (Joined } c)](S) \quad
\end{aligned}
$$

